\title{
FAMILIES OF EXPLICIT ISOGENIES OF HYPERELLIPTIC JACOBIANS
}

\author{
BENJAMIN SMITH
}

\begin{abstract}
We construct three-dimensional families of hyperelliptic curves of genus 6,12 , and 14, two-dimensional families of hyperelliptic curves of genus $3,6,7,10,20$, and 30 , and one-dimensional families of hyperelliptic curves of genus 5, 10 and 15, all of which are equipped with an an explicit isogeny from their Jacobian to another hyperelliptic Jacobian. We show that the Jacobians are generically absolutely simple, and describe the kernels of the isogenies. The families are derived from Cassou-Noguès and Couveignes' explicit classification of pairs $(f, g)$ of polynomials such that $f\left(x_{1}\right)-g\left(x_{2}\right)$ is reducible.
\end{abstract}

\section{INTRODUCTION}

In this article, we construct twelve explicit families of isogenies of hyperelliptic Jacobians. By explicit, we mean that we provide equations for hyperelliptic curves generating the domains and codomains of each isogeny, together with a correspondence on the curves realizing the isogeny as a map on divisor classes. Our main results are summarized by Theorem 1.1, which follows from the examples of $\S 6$. We also construct some families of Jacobians with explicit Real Multiplication, including and generalizing the families described by Tautz, Top, and Verberkmoes [26].

Theorem 1.1. For each row of the following table, there exists an n-dimensional family of explicit isogenies of Jacobians of hyperelliptic curves of genus $g$ over $k$, with kernel isomorphic to $G$ (and hence splitting multiplication-by-m). The generic fibre of each family is an isogeny of absolutely simple Jacobians.

\begin{tabular}{|r|r|r|l|l|}
\hline$g$ & $n$ & {$[m]$} & $G$ & $k$ \\
\hline \hline 3 & 2 & {$[2]$} & $(\mathbb{Z} / 2 \mathbb{Z})^{3}$ & $\mathbb{Q}(\sqrt{-7})$ \\
\hline 5 & 1 & {$[3]$} & $(\mathbb{Z} / 3 \mathbb{Z})^{5}$ & $\mathbb{Q}(\sqrt{-11})$ \\
\hline 6 & 3 & {$[2]$} & $(\mathbb{Z} / 2 \mathbb{Z})^{6}$ & $\mathbb{Q}(\sqrt{-7})$ \\
\hline 6 & 2 & {$[3]$} & $(\mathbb{Z} / 3 \mathbb{Z})^{6}$ & $\mathbb{Q}(\sqrt{-3 \sqrt{13}}+1)$ \\
\hline 7 & 2 & {$[4]$} & $(\mathbb{Z} / 4 \mathbb{Z})^{4} \times(\mathbb{Z} / 2 \mathbb{Z})^{6}$ & $\mathbb{Q}(\sqrt{-15})$ \\
\hline 10 & 2 & {$[3]$} & $(\mathbb{Z} / 3 \mathbb{Z})^{10}$ & $\mathbb{Q}(\sqrt{-11})$ \\
\hline 10 & 1 & {$[4]$} & $(\mathbb{Z} / 4 \mathbb{Z})^{9} \times(\mathbb{Z} / 2 \mathbb{Z})^{2}$ & $\mathbb{Q}(\sqrt{-7})$ \\
\hline 12 & 3 & {$[3]$} & $(\mathbb{Z} / 3 \mathbb{Z})^{12}$ & $\mathbb{Q}(\sqrt{-3 \sqrt{13}}+1)$ \\
\hline 14 & 3 & {$[4]$} & $(\mathbb{Z} / 4 \mathbb{Z})^{9} \times(\mathbb{Z} / 2 \mathbb{Z})^{10}$ & $\mathbb{Q}(\sqrt{-15})$ \\
\hline 15 & 1 & {$[8]$} & $(\mathbb{Z} / 8 \mathbb{Z})^{5} \times(\mathbb{Z} / 4 \mathbb{Z})^{10} \times(\mathbb{Z} / 4 \mathbb{Z})^{10}$ & Sextic CM-field (see Ex. 4.8) \\
\hline 20 & 2 & {$[4]$} & $(\mathbb{Z} / 4 \mathbb{Z})^{19} \times(\mathbb{Z} / 2 \mathbb{Z})^{2}$ & $\mathbb{Q}(\sqrt{-7})$ \\
\hline 30 & 2 & {$[8]$} & $(\mathbb{Z} / 8 \mathbb{Z})^{11} \times(\mathbb{Z} / 4 \mathbb{Z})^{19} \times(\mathbb{Z} / 4 \mathbb{Z})^{19}$ & Sextic CM-field (see Ex. 4.8) \\
\hline
\end{tabular}

Our families of isogenies are derived from the remarkable explicit classification of pairs of polynomials $(f, g)$ such that $f\left(x_{1}\right)-g\left(x_{2}\right)$ is reducible due to CassouNogùes and Couveignes [5], building upon the work of Fried [10, 11, 12], Feit [7, 8, 9], and others [4]. We associate a family of pairs of curves to every such pair $(f, g)$, and a family of explicit homomorphisms (between the Jacobians of the curves of each pair) to each factor of $f\left(x_{1}\right)-g\left(x_{2}\right)$. We show that each homomorphism is 
in fact an isogeny of (generically) absolutely simple Jacobians, and compute the isomorphism type of the kernels. We also calculate the dimension of the image of each family in its appropriate moduli space.

Over the complex field, abelian varieties are complex tori, and we may construct isogenies by working with period matrices. Over arbitrary fields, these methods are not available to us; the only abelian varieties for which we have a convenient representation for explicit computation are Jacobians of curves, where we can use the standard isomorphism with the divisor class group. However, the Jacobians occupy a positive-codimension subspace of the moduli space of abelian varieties in dimension greater than three, so an isogeny with a Jacobian for a domain generally does not have another Jacobian for a codomain. For this reason, examples of explicit isogenies of higher-dimensional abelian varieties are particularly rare (setting aside endomorphisms such as integer multiplication and Frobenius). We note that recently, Mestre has described a $(g+1)$-dimensional family of $(\mathbb{Z} / 2 \mathbb{Z})^{g}$-isogenies of Jacobians of hyperelliptic curves of genus $g$ for every $g \geq 1$ (see [21]). Our families of isogenies are defined over number fields, and provide a source of examples of explicit isogenies of high-dimensional abelian varieties over exact fields.

This work generalizes some results from the author's unpublished thesis $[24, \S 6]$. The subfamily at $s=0$ of the isogeny in Example 6.1 and the fibre at $s=0$ of the isogeny in Example 6.3 also appeared earlier in the thesis of Kux [18, §4.1]. The Real Multiplication families in Examples 5.2 and 5.3 appeared in the work of Tautz, Top, and Verberkmoes [26]. We assume some familiarity with the basic theory of curves and abelian varieties, referring the reader to Birkenhake and Lange [1], Hindry and Silverman [14, Part A], Milne [22], and Shimura [23] for further details.

Notation. Throughout this article, $\zeta_{n}$ denotes a primitive $n^{\text {th }}$ root of unity in $\overline{\mathbb{Q}}$. If $\sigma$ is an automorphism of a field $k$ and $f(x)=\sum_{i} c_{i} x^{i}$ is a polynomial over $k$, then we write $f^{\sigma}(x)$ for the polynomial $\sum_{i} c_{i}^{\sigma} x^{i}$. If $\phi$ is an isogeny of abelian varieties with kernel isomorphic to a group $G$, then we say that $\phi$ is a $G$-isogeny.

Acknowledgements. The author is grateful to the mathematics departments of the University of Sydney and Royal Holloway, University of London, where parts of this work were carried out. This research was supported in part by EPSRC grant $\mathrm{EP} / \mathrm{C} 014839 / 1$.

\section{The BAsic CONSTRUCtion}

Suppose $(f, g)$ is a pair of squarefree polynomials of degree at least 5 over $k$ such that there exists a nontrivial factorization

$$
f\left(x_{1}\right)-g\left(x_{2}\right)=A\left(x_{1}, x_{2}\right) B\left(x_{1}, x_{2}\right) .
$$

Given such a pair of polynomials, we define a pair $(X, Y)$ of hyperelliptic curves by

$$
X: y_{1}^{2}=f\left(x_{1}\right) \quad \text { and } \quad Y: y_{2}^{2}=g\left(x_{2}\right) .
$$

The factors $A$ and $B$ of $f\left(x_{1}\right)-g\left(x_{2}\right)$ define explicit homomorphisms from $J_{X}$ to $J_{Y}$ as follows: Let $C$ be the correspondence on $X \times Y$ defined by

$$
C:=V\left(y_{1}-y_{2}, A\left(x_{1}, x_{2}\right)\right) \subset X \times Y .
$$

The natural projections of $X \times Y$ restrict to coverings $\pi_{X}: C \rightarrow X$ and $\pi_{Y}: C \rightarrow Y$. Composing the pullback $\left(\pi_{X}\right)^{*}: J_{X} \rightarrow J_{C}$ with the pushforward $\left(\pi_{Y}\right)_{*}: J_{C} \rightarrow J_{Y}$, we obtain a homomorphism

$$
\phi:=\left(\pi_{Y}\right)_{*} \circ\left(\pi_{X}\right)^{*}: J_{X} \longrightarrow J_{Y}
$$


we say $\phi$ is induced by $C$. The homomorphism $\phi$ is completely explicit: we can compute the image of a divisor class on $X$ under $\phi$ by pulling back a representative divisor to $C$ and then pushing the result forward onto $Y$.

If we replace $A$ with $B$ in (1), we obtain the homomorphism $-\phi$. Exchanging $X$ and $Y$ in (2), we obtain the Rosati dual homomorphism $\phi^{\dagger}: J_{Y} \rightarrow J_{X}$ (recall $\phi^{\dagger}=\lambda_{X}^{-1} \hat{\phi} \lambda_{Y}$, where $\hat{\phi}: \widehat{J}_{Y} \rightarrow \widehat{J}_{X}$ is the dual homomorphism and $\lambda_{X}: J_{X} \stackrel{\sim}{\rightarrow} \widehat{J}_{X}$ and $\lambda_{Y}: J_{Y} \stackrel{\sim}{\rightarrow} \widehat{J}_{Y}$ are the canonical principal polarizations).

If $A\left(x_{1}, x_{2}\right)$ divides $f\left(x_{1}\right)-g\left(x_{2}\right)$, then it also divides $F\left(f\left(x_{1}\right)\right)-F\left(g\left(x_{2}\right)\right)$ for every polynomial $F$ over $k$. Therefore, if we let $F=x^{d}+s_{1} x^{d-1}+\cdots+s_{d-1} x+s_{d}$ be the generic monic polynomial of degree $d$ (where the $s_{i}$ are free parameters), let $\Delta_{f}$ (resp. $\Delta_{g}$ ) be the discriminant of $F(f(x))$ (resp. $\left.F(g(x))\right)$, and let $T$ be the parameter space defined by

$$
T:=\operatorname{Spec}\left(k\left[s_{1}, \ldots, s_{d}\right]\right) \backslash\left(V\left(\Delta_{f}\right) \cup V\left(\Delta_{g}\right)\right),
$$

then we obtain a $d$-parameter family $(\mathcal{X}, \mathcal{Y}) \rightarrow T$ of pairs of curves defined by

$$
\mathcal{X}: y_{1}^{2}=F\left(f\left(x_{1}\right)\right)=f\left(x_{1}\right)^{d}+s_{1} f\left(x_{1}\right)^{d-1}+\cdots+s_{d-1} f\left(x_{1}\right)+s_{d}
$$

and

$$
\mathcal{Y}: y_{2}^{2}=F\left(g\left(x_{2}\right)\right)=g\left(x_{2}\right)^{d}+s_{1} g\left(x_{2}\right)^{d-1}+\cdots+s_{d-1} g\left(x_{2}\right)+s_{d},
$$

together with a family of homomorphisms $\phi: \mathcal{J}_{\mathcal{X}} \rightarrow \mathcal{J}_{\mathcal{Y}}$ induced by the correspondence

$$
C=V\left(y_{1}-y_{2}, A\left(x_{1}, x_{2}\right)\right) \subset \mathcal{X} \times_{T} \mathcal{Y}
$$

That is, for each $P$ in $T$, if $C_{P}, X_{P}$, and $Y_{P}$ are the fibres of $C, \mathcal{X}$, and $\mathcal{Y}$ over $P$, then $C_{P}$ induces a homomorphism $\phi_{P}: J_{X_{P}} \rightarrow J_{Y_{P}}$.

If $f$ and $g$ are defined over a polynomial ring $k[t]$, then we can define $(d+1)$ parameter families of pairs of curves and homomorphisms, this time parameterised by $T=\operatorname{Spec}\left(k\left[t, s_{1}, \ldots, s_{d}\right]\right) \backslash\left(V\left(\Delta_{f}\right) \cup V\left(\Delta_{g}\right)\right)$, in exactly the same way. Throughout this article we will use $T$ to denote the parameter space of each of our families; the precise definition of $T$ in each case will be clear from the context.

We will restrict our attention to the cases $d=\operatorname{deg} F=1$ (the linear construction) and $d=\operatorname{deg} F=2$ (the quadratic construction). For higher degrees $d$, the Jacobians of $\mathcal{X}$ and $\mathcal{Y}$ are reducible. Indeed, we have a covering $(x, y) \mapsto(f(x), y)$ from $\mathcal{X}$ to the curve $\mathcal{X}^{\prime}: v^{2}=F(u)$, so $\mathcal{J}_{\mathcal{X}}$ is an isogeny factor of $\mathcal{J}_{\mathcal{X}}$ whenever $\mathcal{X}^{\prime}$ has positive genus: that is, whenever $d>2$. We aim to construct explicit isogenies of absolutely simple Jacobians, so we will leave aside $d>2$.

Remark 2.1. Our constructions depend only on $f$ and $g$, and generalize to variableseparated curves - that is, curves of the form $P(y)=f(x)$ where $\operatorname{deg} P>2$. The analysis of the resulting homomorphisms is more detailed, however, and some of the methods we use in $\S 3$ do not readily extend to the separated-variable case. We will return to these constructions in future work.

\section{Determining Kernel Structure}

Suppose $\mathcal{X}, \mathcal{Y}$, and $\phi: \mathcal{J}_{\mathcal{X}} \rightarrow \mathcal{J}_{\mathcal{Y}}$ are defined as in the previous section. We want to determine whether $\phi$ is an isogeny, and if so to compute a group $G$ isomorphic to its kernel. It suffices to consider the generic fibre $\phi: J_{X} \rightarrow J_{Y}$, which is defined over $\overline{\mathbb{Q}}(T)$, a field of characteristic zero.

The first step is to show that $J_{X}$ is absolutely simple; then $\phi$ is an isogeny if and only if it is nonzero. Further, if $\phi$ is an isogeny and $J_{X}$ is absolutely simple and $g_{X}=g_{Y}$ then $J_{Y}$ must also be absolutely simple, and $\phi$ itself cannot arise from a product of isogenies of lower-dimensional abelian varieties. Since a reducible abelian variety cannot specialize to to an absolutely simple one, it is enough to exhibit a point $P$ of the parameter space $T$ such that the specialization $J_{P}$ of $\mathcal{J}_{\mathcal{X}}$ 
at $P$ is absolutely simple. In Examples 6.1 and 6.5, there will exist a convenient choice of $P$ allowing us to deduce the simplicity of $J_{P}$ from CM-theory. For the other examples, we will use the fact that $J_{P}$ is defined over a number field $K$, and exhibit a prime $\mathfrak{p}$ of $K$ such that the (good) reduction $\overline{J_{P}}$ of $J_{P}$ at $\mathfrak{p}$ is absolutely simple; the absolute simplicity of $J_{P}$, and thus the absolute simplicity of $J_{X}$, then follows from [6, Lemma 6].

To show that $\overline{J_{P}}$ is absolutely simple, we compute its Weil polynomial $\chi$ (that is, the characteristic polynomial of its Frobenius endomorphism) using Kedlaya's algorithm [16], which is implemented in Magma [13, 2]. (For this to be practical the norm of $\mathfrak{p}$ must be a power of a small prime, especially for the higher-genus families.) If $\chi$ is irreducible, then $\overline{J_{P}}$ is simple. To determine whether $\overline{J_{P}}$ is absolutely simple, we apply the criterion appearing in $[15]$ :

Lemma 3.1 (Howe and Zhu). Suppose $A$ is a simple abelian variety over a finite field, and let $\chi$ be its (irreducible) Weil polynomial. Let $\pi$ be an element of $\overline{\mathbb{Q}}$ satisfying $\chi(\pi)=0$. Let $D$ be the set of integers $d>1$ such that either

(1) $\chi(x)$ lies in $\mathbb{Z}\left[x^{d}\right]$, or

(2) $\left[\mathbb{Q}(\pi): \mathbb{Q}\left(\pi^{d}\right)\right]>1$ and $\mathbb{Q}(\pi)=\mathbb{Q}\left(\pi^{d}, \zeta_{d}\right)$.

If $D$ is empty, then $A$ is absolutely simple.

Proof. See [15, Proposition 3]. Note that $D \subset\left\{d \in \mathbb{Z}_{>0}: \varphi(d) \mid 2 \operatorname{dim} A\right\}$, so this criterion can be efficiently checked.

We will be handling some large Weil polynomials. To save space, we will use the following, more compact representation.

Definition 3.1. Suppose $A$ is a $g$-dimensional abelian variety over $\mathbb{F}_{q}$ with Weil polynomial $\chi$. We define the Weil coefficients of $A$ to be the integers $w_{1}, \ldots, w_{g}$ such that

$$
\chi(x)=x^{2 g}+w_{1} t^{2 g-1}+\cdots+w_{g} x^{g}+w_{g-1} q x^{g-1}+\cdots+w_{1} q^{g-1} x+q^{g} .
$$

Recall that $\phi^{\dagger} \circ \phi$ is an endomorphism of $\mathcal{J}_{\mathcal{X}}$; if $\phi$ is an isogeny of absolutely simple Jacobians, then $\phi^{\dagger} \circ \phi=[m]_{\mathcal{J}}$ for some nonzero integer $m$. Conversely, if $\phi^{\dagger} \circ \phi=[m]_{\mathcal{J}_{\mathcal{X}}}$ for some $m$, then $\phi$ is an isogeny and $\operatorname{ker} \phi \subset \mathcal{J}_{\mathcal{X}}[m]$. Since $\phi$ is an isogeny of Jacobians (thus respecting the canonical polarizations), its kernel must be a maximal isotropic subgroup of $\mathcal{J}_{\mathcal{X}}[\mathrm{m}]$ with respect to the $m$-Weil pairing; the nondegeneracy of the Weil pairing then gives the following elementary result.

Lemma 3.2. If $\phi^{\dagger} \circ \phi=[m]_{\mathcal{J}}$ for some positive integer $m$, then $\phi$ is a $G$-isogeny for some subgroup $G$ of $(\mathbb{Z} / m \mathbb{Z})^{2 g_{X}}$ such that $G \cong(\mathbb{Z} / m \mathbb{Z})^{2 g_{X}} / G$. Further, if $m$ is squarefree, then $G \cong(\mathbb{Z} / m \mathbb{Z})^{g_{X}}$.

Let $K=\overline{\mathbb{Q}}(T)$ denote the base field of the generic fibre, and let $\Omega(X)$ and $\Omega(Y)$ denote the $K$-vector spaces of regular differentials on $X$ and $Y$, respectively. We have the well-known representation

$$
D_{X, Y}(\cdot): \operatorname{Hom}\left(J_{X}, J_{Y}\right) \longrightarrow \operatorname{Hom}(\Omega(X), \Omega(Y)),
$$

sending a homomorphism to the induced map on differentials (see Shimura [23, §2.9] for details). This representation is faithful in characteristic zero, and it respects composition: if $\phi: J_{X} \rightarrow J_{Y}$ and $\psi: J_{Y} \rightarrow J_{Z}$ are homomorphisms, then

$$
D_{X, Z}(\psi \circ \phi)=D_{X, Y}(\phi) D_{Y, Z}(\psi) \text {. }
$$

In particular, when $J_{X} \cong J_{Y}$ we obtain a representation of rings

$$
D_{X}(\cdot): \operatorname{End}\left(J_{X}\right) \longrightarrow \operatorname{End}(\Omega(X)) \text {. }
$$


To determine whether $\phi$ is an isogeny, we compute $D_{X}\left(\phi^{\dagger} \phi\right)=D_{X, Y}(\phi) D_{Y, X}\left(\phi^{\dagger}\right)$ and check that the result is equal to $m I_{g_{X}}$ for some integer $m \neq 0$. Given $m$, we can use Lemma 3.2 to partially determine the group structure of ker $\phi$.

It is straightforward to compute $D_{X, Y}(\phi)$ when $\phi$ is induced by a correspondence of the form $C=V\left(y_{1}-y_{2}, A\left(x_{1}, x_{2}\right)\right) \subset X \times Y$. We begin by fixing ordered bases

$$
\Omega(X)=\left\langle d\left(x_{1}^{i}\right) / y_{1}: 1 \leq i \leq g_{X}\right\rangle \quad \text { and } \quad \Omega(Y)=\left\langle d\left(x_{2}^{i}\right) / y_{2}: 1 \leq i \leq g_{Y}\right\rangle
$$

for $\Omega(X)$ and $\Omega(Y)$. Then $D_{X, Y}(\cdot)$ becomes a representation into $\operatorname{Mat}_{g_{X} \times g_{Y}}(K)$ (viewing elements of $\Omega(X)$ and $\Omega(Y)$ as row vectors, with matrices representing homomorphisms act by multiplication on the right.) Pulling back our basis of $\Omega(X)$ to $\Omega(C)$ (via the inclusion $K(X) \hookrightarrow K(C)$ induced by $\pi_{X}$ ) and then taking the trace from $\Omega(C)$ to $\Omega(Y)$ (with respect to the inclusion $K(Y) \hookrightarrow K(C)$ induced by $\pi_{Y}$ ), we have

$$
\phi_{*}\left(d\left(x_{1}^{i}\right) / y_{1}\right)=\operatorname{Tr}_{\Omega(Y)}^{\Omega(C)}\left(d\left(x_{1}^{i}\right) / y_{1}\right)=d t_{i} / y_{2},
$$

where $t_{i}$ is the trace from $K(C)$ to $K(Y)$ of $x_{1}^{i}$. To compute these traces, we rewrite $A\left(x_{1}, x_{2}\right)$ as a polynomial in $x_{1}$ over $K\left[x_{2}\right]$ (after possibly rescaling to ensure $A$ is monic in $\left.x_{1}\right)$ :

$$
A\left(x_{1}, x_{2}\right)=x_{1}^{d}+\sum_{i=1}^{d}(-1)^{i} s_{i}\left(x_{2}\right) x_{1}^{d-i} .
$$

The $s_{i}$ are the $i^{\text {th }}$ elementary symmetric polynomials in the roots of $A$ viewed as a polynomial in $x_{1}$ over $K\left(x_{2}\right)$; each $s_{i}$ is a polynomial in $x_{2}$ over $K$ of degree at most $i$. The function $t_{i}$ is by definition the $i^{\text {th }}$ power sum symmetric function in these same roots, and so we can express the $t_{i}$ in terms of the $s_{j}$ using the standard Newton-Girard recurrences:

$$
k s_{k}=\sum_{i=1}^{k}(-1)^{i-1} s_{k-i} t_{i} .
$$

Since each $s_{i}$ has degree at most $i$, it follows that each of the trace functions $t_{i}$ has degree at most $i$. We can therefore write

$$
d\left(t_{i}\right) / y_{2}=\sum_{j=1}^{i} t_{i, j} d\left(x_{2}^{j}\right) / y_{2}
$$

with coefficients $t_{i, j}$ in $K$; these coefficients are precisely the entries of $D_{X, Y}(\phi)$ (with $t_{i, j}=0$ for $j>i$ ).

We noted above that if $\phi: J_{X} \rightarrow J_{Y}$ is a homomorphism induced by a correspondence on $X \times Y$, then we obtain the Rosati dual $\phi^{\dagger}: J_{Y} \rightarrow J_{X}$ by simply exchanging $X$ and $Y$. We may therefore compute $D_{Y, X}\left(\phi^{\dagger}\right)$ in exactly the same way we computed $D_{X, Y}(\phi)$, expressing the differentials $\phi_{*}^{\dagger}\left(d\left(x_{2}^{i}\right) / y_{2}\right)=\operatorname{Tr}_{\Omega(X)}^{\Omega(C)}\left(d\left(x_{2}^{i}\right) / y_{2}\right)$ as linear combinations of the $d\left(x_{1}^{j}\right) / y_{1}$.

If $\phi^{\dagger} \circ \phi=[m]_{J_{X}}$, then Lemma 3.2 allows us to determine the structure of ker $\phi$ when $m$ is squarefree. But in $\S 6$ we will encounter $m=2,3,4$, and 8; we will therefore need another technique to handle $m=4$ and $m=8$.

Lemma 3.3. Let $\phi: J_{X} \rightarrow J_{Y}$ be an isogeny over a field of characteristic not 2, such that $\phi^{\dagger} \phi=[m]_{X}$ with $m=4$ or 8 , and let $\nu$ be the $(\mathbb{Z} / 2 \mathbb{Z})$-rank of $\operatorname{ker} \phi \cap J_{X}[2]$.

(1) If $m=4$, then $\operatorname{ker} \phi \cong(\mathbb{Z} / 4 \mathbb{Z})^{2 g_{X}-\nu} \times(\mathbb{Z} / 2 \mathbb{Z})^{2\left(\nu-g_{X}\right)}$.

(2) If $m=8$, then $\operatorname{ker} \phi \cong(\mathbb{Z} / 8 \mathbb{Z})^{2 g_{X}-\nu} \times(\mathbb{Z} / 4 \mathbb{Z})^{\nu-g_{X}} \times(\mathbb{Z} / 4 \mathbb{Z})^{\nu-g_{X}}$.

Proof. The result follows directly from Lemma 3.2.

To apply Lemma 3.3, we need to compute the $(\mathbb{Z} / 2 \mathbb{Z})$-rank $\nu$ of $\operatorname{ker} \phi \cap J_{X}[2]$. 
Lemma 3.4. Let $f(x)=\prod_{i=1}^{d}\left(x-\gamma_{i}\right)$ and $g(x)=\prod_{i=1}^{d}\left(x-\delta_{i}\right)$ be polynomials of degree $d>2$ over a field of characteristic not 2 such that $f\left(x_{1}\right)-g\left(x_{2}\right)$ has a nontrivial factor $A\left(x_{1}, x_{2}\right)$. Let $X: y_{1}^{2}=f\left(x_{1}\right)$ and $Y: y_{2}^{2}=g\left(x_{2}\right)$ be hyperelliptic curves, and $\phi: J_{X} \rightarrow J_{Y}$ the homomorphism induced by the correspondence $V\left(y_{1}-y_{2}, A\left(x_{1}, x_{2}\right)\right)$ on $X \times Y$. The $(\mathbb{Z} / 2 \mathbb{Z})$-rank of $\operatorname{ker} \phi \cap J_{X}[2]$ is given by

$$
\operatorname{rank}_{(\mathbb{Z} / 2 \mathbb{Z})}\left(\operatorname{ker} \phi \cap J_{X}[2]\right)=\operatorname{dim}(\operatorname{ker} M),
$$

where $M$ is the $2 g_{X} \times 2 g_{Y}$ matrix over $\mathbb{F}_{2}$ with $i, j$-th entry $\nu_{i, j}+\nu_{i, 2 g_{Y}+1}(\bmod 2)$, where $\nu_{i, j}$ denotes the multiplicity of $\left(x_{2}-\delta_{j}\right)$ as factor of $A\left(\gamma_{i}, x_{2}\right)$ for $1 \leq i, j \leq d$.

Proof. For each $1 \leq i \leq d$, we let $w_{i}$ be the point $\left(\gamma_{i}, 0\right)$ on $X$ and let $w_{i}^{\prime}$ be the point $\left(\delta_{i}, 0\right)$ on $Y$. If $d$ is odd (so $d=2 g_{X}+1=2 g_{Y}+1$ ), then we let $w_{2 g_{X}+2}$ (resp. $w_{2 g_{Y}+2}^{\prime}$ ) be the unique point at infinity on $X$ (resp. $Y$ ), and we set $\nu_{i, 2 g_{Y}+2}:=0$ for $1 \leq i \leq 2 g_{X}$. The sets $\left\{w_{i}: 1 \leq i \leq 2 g_{X}+2\right\}$ and $\left\{w_{i}^{\prime}: 1 \leq i \leq 2 g_{X}+2\right\}$ are then the sets of Weierstrass points of $X$ and $Y$, respectively. It is well-known that $J_{X}[2]$ (resp. $J_{Y}[2]$ ) is generated by differences of Weierstrass points of $X$ (resp. $Y$ ), subject to the relations

$$
\begin{aligned}
& {\left[\left(w_{2 g_{X}+1}\right)-\left(w_{2 g_{X}+2}\right)\right]=\sum_{i=1}^{2 g_{X}}\left[\left(w_{i}\right)-\left(w_{2 g_{X}+2}\right)\right] \quad \text { and }} \\
& {\left[\left(w_{2 g_{Y}+1}^{\prime}\right)-\left(w_{2 g_{Y}+2}^{\prime}\right)\right]=\sum_{i=1}^{2 g_{Y}}\left[\left(w_{i}^{\prime}\right)-\left(w_{2 g_{Y}+2}^{\prime}\right)\right] .}
\end{aligned}
$$

We therefore fix explicit bases for the 2-torsion:

$$
J_{X}[2]=\left\langle\left[\left(w_{i}\right)-\left(w_{2 g_{X}+2}\right)\right]\right\rangle_{i=1}^{2 g_{X}} \quad \text { and } \quad J_{Y}[2]=\left\langle\left[\left(w_{i}^{\prime}\right)-\left(w_{2 g_{Y}+2}^{\prime}\right)\right]\right\rangle_{i=1}^{2 g_{Y}} .
$$

Since $\phi$ restricts to a homomorphism $\left.\phi\right|_{2}: J_{X}[2] \rightarrow J_{Y}[2]$, we have a representation

$$
T_{2}(\cdot): \operatorname{Hom}\left(J_{X}, J_{Y}\right) \longrightarrow \operatorname{Hom}\left(J_{X}[2], J_{Y}[2]\right) \cong \operatorname{Mat}_{2 g_{X} \times 2 g_{Y}}\left(\mathbb{F}_{2}\right)
$$

(where the isomorphism is determined by our choice of bases.) The $(\mathbb{Z} / 2 \mathbb{Z})$-rank of $\operatorname{ker} \phi \cap J_{X}[2]=\left.\operatorname{ker} \phi\right|_{2}$ is then equal to the nullity of the matrix $T_{2}(\phi)$. The entries $t_{i, j}$ of $T_{2}(\phi)$ are determined by the relations

$$
\phi\left(\left[\left(w_{i}\right)-\left(w_{2 g_{X}+2}\right)\right]\right)=\sum_{j=1}^{2 g_{Y}} t_{i, j}\left[\left(w_{j}^{\prime}\right)-\left(w_{2 g_{Y}+2}^{\prime}\right)\right]
$$

(this is well-defined, since the $t_{i, j}$ are elements of $\mathbb{Z} / 2 \mathbb{Z}$ ). Explicitly computing the images of the basis elements, we find

$$
\begin{aligned}
\phi\left(\left[\left(w_{i}\right)-\left(w_{2 g_{X}+2}\right)\right]\right)= & \sum_{j=1}^{2 g_{Y}+1}\left(\nu_{i, j}-\nu_{i, 2 g_{Y}+2}\right)\left[\left(w_{i}^{\prime}\right)-\left(w_{2 g_{Y}+2}^{\prime}\right)\right] \\
= & \left(\sum_{j=1}^{2 g_{Y}}\left(\nu_{i, j}-\nu_{i, 2 g_{Y}+2}\right)\left[\left(w_{i}^{\prime}\right)-\left(w_{2 g_{Y}+2}^{\prime}\right)\right]\right) \\
& +\left(\nu_{i, 2 g_{Y}+1}-\nu_{i, 2 g_{Y}+2}\right)\left(\sum_{j=1}^{2 g_{Y}}\left[\left(w_{i}^{\prime}\right)-\left(w_{2 g+2}^{\prime}\right)\right]\right) \\
= & \sum_{j=1}^{2 g_{Y}}\left(\nu_{i, j}+\nu_{i, 2 g_{Y}+1}-2 \nu_{i, 2 g_{Y}+2}\right)\left[\left(w_{i}^{\prime}\right)-\left(w_{2 g_{Y}+2}^{\prime}\right)\right] \\
= & \sum_{j=1}^{2 g_{g_{Y}}}\left(\nu_{i, j}+\nu_{i, 2 g_{Y}+1}\right)\left[\left(w_{i}^{\prime}\right)-\left(w_{2 g_{Y}+2}^{\prime}\right)\right]
\end{aligned}
$$

so $t_{i, j} \equiv \nu_{i, j}+\nu_{i, 2 g+1}(\bmod 2)$ for $1 \leq j \leq 2 g_{Y}$ and $1 \leq i \leq 2 g_{X}$. Hence $M=T_{2}(\phi)$, and the result follows.

Remark 3.1. In practice, computing the matrix $M$ of Lemma 3.4 can be difficult if the roots of $f$ and $g$ are not all defined over a low-degree extension of the ground field. In our examples, we will be free to choose (reductions of) $X$ and $Y$ in such a way that all of the roots of $f$ and $g$ lie in a small finite field. 


\section{PAirs of POlynomials}

In order to use the construction of $\S 2$ to produce examples of explicit isogenies, we need a source of pairs of polynomials $(f, g)$ such that $f\left(x_{1}\right)-g\left(x_{2}\right)$ is reducible. We will use the explicit classification of such pairs over $\mathbb{C}$ due to Cassou-Noguès and Couveignes [5], which we summarize in Theorem 4.1. This classification is restricted to indecomposable polynomials (in the sense of Definition 4.2), and classifies pairs up to an equivalence relation described in Definition 4.1 .

Definition 4.1. We say that polynomials $f_{1}$ and $f_{2}$ over $k$ are linear translates if there exist $a$ and $b$ in $\bar{k}$, with $a \neq 0$, such that $f_{1}(x)=f_{2}(a x+b)$. We say pairs $\left(f_{1}, g_{1}\right)$ and $\left(f_{2}, g_{2}\right)$ of polynomials are equivalent if there exists $c \neq 0$ and $d$ in $\bar{k}$ such that $f_{1}$ and $c f_{2}+d$ are linear translates and $g_{1}$ and $c g_{2}+d$ are linear translates.

The "equivalence" of Definition 4.1 is indeed an equivalence relation on pairs of polynomials. Further, if $\mathcal{S}$ is an equivalence class, then either $f\left(x_{1}\right)-g\left(x_{2}\right)$ is $\bar{k}$-reducible for each $(f, g)$ in $\mathcal{S}$ or $f\left(x_{1}\right)-g\left(x_{2}\right)$ is $\bar{k}$-irreducible for each $(f, g)$ in $\mathcal{S}$.

Definition 4.2. A polynomial $f$ is decomposable if $f(x)=f_{1}\left(f_{2}(x)\right)$ for some polynomials $f_{1}$ and $f_{2}$ of degree at least 2 , and indecomposable otherwise.

Theorem 4.1 (Cassou-Noguès and Couveignes [5]). Let $(f, g)$ be a pair of indecomposable polynomials of degree at least 3 over $\mathbb{C}$, and let $\sigma$ denote complex conjugation. Assume the classification of finite simple groups.

If $f$ and $g$ are linear translates, then $f\left(x_{1}\right)-g\left(x_{2}\right)$ is divisible by $x_{1}-x_{2}$, and $\left(f\left(x_{1}\right)-g\left(x_{2}\right)\right) /\left(x_{1}-x_{2}\right)$ is reducible if and only if $(f, g)$ is equivalent to either

(1) the pair $\left(x^{n}, x^{n}\right)$ for some prime $n$, or

(2) the pair $\left(D_{n}(x), D_{n}(x)\right)$ for some prime $n$, where $D_{n}(x)$ is defined in Example 4.2.

If $f$ and $g$ are not linear translates, then $f\left(x_{1}\right)-g\left(x_{2}\right)$ is reducible if and only if $(f, g)$ is equivalent to one of the following (possibly after exchanging $f$ and $g$ ):

(3) a pair in the one-parameter family $\left(f_{7}, f_{7}^{\sigma}\right)$ defined in Example 4.3, or

(4) the pair $\left(f_{11}, f_{11}^{\sigma}\right)$ defined in Example 4.4, or

(5) a pair in the one-parameter family $\left(f_{13}, f_{13}^{\sigma}\right)$ defined in Example 4.5, or

(6) a pair in the one-parameter family $\left(f_{15},-f_{15}^{\sigma}\right)$ defined in Example 4.6, or

(7) the pair $\left(f_{21}, f_{21}^{\sigma}\right)$ defined in Example 4.7, or

(8) the pair $\left(f_{31}, f_{31}^{\sigma}\right)$ defined in Example 4.8.

Example 4.1 (Cyclic polynomials). The difference $x_{1}^{n}-x_{2}^{n}$ factors as

$$
x_{1}^{n}-x_{2}^{n}=\prod_{e=0}^{n-1}\left(x_{1}-\zeta_{n}^{e} x_{2}\right) .
$$

Example 4.2 (Dickson polynomials). For each $n \geq 1$, we let $D_{n}(x)=D_{n}(x, 1)$ denote the $n^{\text {th }}$ Dickson polynomial of the first kind with parameter 1: that is, the unique polynomial of degree $n$ such that $D_{n}\left(x+x^{-1}, 1\right)=x^{n}+x^{-n}$. In characteristic zero we have $D_{n}(x)=2 T_{n}(x / 2)$, where $T_{n}$ is the classical Chebyshev polynomial of degree $n$. (See [20] for further details.) We have a nontrivial factorization

$$
D_{n}\left(x_{1}\right)-D_{n}\left(x_{2}\right)=\left(x_{1}-x_{2}\right) \prod_{i=1}^{(n-1) / 2} A_{n, i}\left(x_{1}, x_{2}\right)
$$

(see [20, Theorem 3.12]), where

$$
A_{n, i}\left(x_{1}, x_{2}\right):=x_{1}^{2}+x_{2}^{2}-\left(\zeta_{n}^{i}+\zeta_{n}^{-i}\right) x_{1} x_{2}+\left(\zeta_{n}^{i}-\zeta_{n}^{-i}\right) .
$$


Example 4.3 (Polynomials of degree 7). Let $\alpha_{7}$ be an element of $\overline{\mathbb{Q}}$ satisfying

$$
\alpha_{7}^{2}+\alpha_{7}+2=0
$$

The involution $\sigma: \alpha_{7} \mapsto 2 / \alpha_{7}$ generates $\operatorname{Gal}\left(\mathbb{Q}\left(\alpha_{7}\right) / \mathbb{Q}\right)$. Note that $\mathbb{Q}\left(\alpha_{7}\right)=$ $\mathbb{Q}(\sqrt{-7})$ is a quadratic imaginary field, and $\sigma$ acts as complex conjugation.

Let $f_{7}$ be the polynomial of degree 7 over $\mathbb{Q}\left(\alpha_{7}\right)[t]$ defined by

$$
\begin{aligned}
f_{7}(x):= & \frac{1}{7} x^{7}-\alpha_{7} t x^{5}-\alpha_{7} t x^{4}-\left(2 \alpha_{7}+5\right) t^{2} x^{3}-\left(4 \alpha_{7}+6\right) t^{2} x^{2} \\
& +\left(\left(3 \alpha_{7}-2\right) t^{3}-\left(\alpha_{7}+3\right) t^{2}\right) x+\alpha_{7} t^{3} .
\end{aligned}
$$

(Our $f_{7}$ is the polynomial of $[5, \S 5.1]$ with $a_{2}=\alpha_{7}$.) We have a nontrivial factorization $f_{7}\left(x_{1}\right)-f_{7}^{\sigma}\left(x_{2}\right)=A_{7}\left(x_{1}, x_{2}\right) B_{7}\left(x_{1}, x_{2}\right)$, where

$$
A_{7}=x_{1}^{3}-x_{2}^{3}-\alpha_{7}^{\sigma} x_{1}^{2} x_{2}+\alpha_{7} x_{1} x_{2}^{2}+\left(3-2 \alpha_{7}^{\sigma}\right) t x_{1}-\left(3-2 \alpha_{7}\right) t x_{2}+\left(\alpha_{7}-\alpha_{7}^{\sigma}\right) t ;
$$

note that $A_{7}\left(x_{2}, x_{1}\right)=-A_{7}\left(x_{1}, x_{2}\right)^{\sigma}$. Both $A_{7}$ and $B_{7}$ are absolutely irreducible.

Example 4.4 (Polynomials of degree 11). Let $\alpha_{11}$ be an element of $\overline{\mathbb{Q}}$ satisfying

$$
\alpha_{11}^{2}+\alpha_{11}+3=0
$$

the involution $\sigma: \alpha_{11} \mapsto 3 / \alpha_{11}$ generates $\operatorname{Gal}\left(\mathbb{Q}\left(\alpha_{11}\right) / \mathbb{Q}\right)$. Note that $\mathbb{Q}\left(\alpha_{11}\right)=$ $\mathbb{Q}(\sqrt{-11})$ is an imaginary quadratic field, and $\sigma$ acts as complex conjugation.

Let $f_{11}$ be the polynomial of degree 11 over $\mathbb{Q}\left(\alpha_{11}\right)$ defined by

$$
\begin{aligned}
f_{11}(x):= & \frac{1}{11} x^{11}+\alpha_{11} x^{9}+2 x^{8}-3\left(\alpha_{11}+4\right) x^{7}+16 \alpha_{11} x^{6} \\
& -3\left(7 \alpha_{11}-5\right) x^{5}-30\left(\alpha_{11}+4\right) x^{4}+63\left(\alpha_{11}+1\right) x^{3} \\
& -20\left(5 \alpha_{11}-1\right) x^{2}-3\left(8 \alpha_{11}+47\right) x+18 \alpha_{11} .
\end{aligned}
$$

(Our $f_{11}$ is the polynomial of $[5, \S 5.2]$ with $a_{2}=\alpha_{11}^{\sigma}$.) We have a nontrivial factorization $f_{11}\left(x_{1}\right)-f_{11}^{\sigma}\left(x_{2}\right)=A_{11}\left(x_{1}, x_{2}\right) B_{11}\left(x_{1}, x_{2}\right)$, where

$$
\begin{aligned}
A_{11}\left(x_{1}, x_{2}\right)= & x_{1}^{5}-\alpha_{11} x_{1}^{4} x_{2}-x_{1}^{3} x_{2}^{2}+\left(4 \alpha_{11}+2\right) x_{1}^{3}+x_{1}^{2} x_{2}^{3}+\left(\alpha_{11}+6\right) x_{1}^{2} x_{2} \\
& -\left(2 \alpha_{11}-10\right) x_{1}^{2}-\left(\alpha_{11}+1\right) x_{1} x_{2}^{4}+\left(\alpha_{11}-5\right) x_{1} x_{2}^{2} \\
& -\left(12 \alpha_{11}+6\right) x_{1} x_{2}+\left(8 \alpha_{11}-7\right) x_{1}-x_{2}^{5}+\left(4 \alpha_{11}+2\right) x_{2}^{3} \\
& -\left(2 \alpha_{11}+12\right) x_{2}^{2}+\left(8 \alpha_{11}+15\right) x_{2}+12 \alpha_{11}+6 ;
\end{aligned}
$$

note that $A_{11}\left(x_{2}, x_{1}\right)=-A_{11}^{\sigma}\left(x_{1}, x_{2}\right)$. Both $A_{11}$ and $B_{11}$ are absolutely irreducible.

Example 4.5 (Polynomials of degree 13). Let $\beta_{13}$ and $\alpha_{13}$ be elements of $\overline{\mathbb{Q}}$ satisfying

$$
\beta_{13}^{2}-5 \beta_{13}+3=0 \quad \text { and } \quad \alpha_{13}^{2}+\left(\beta_{13}-2\right) \alpha_{13}+\beta_{13}=0 .
$$

The involution $\sigma: \alpha_{13} \mapsto \beta_{13} / \alpha_{13}$ generates $\operatorname{Gal}\left(\mathbb{Q}\left(\alpha_{13}\right) / \mathbb{Q}\left(\beta_{13}\right)\right)$. Observe that $\mathbb{Q}\left(\beta_{13}\right)=\mathbb{Q}(\sqrt{13})$ is a real quadratic field, and $\mathbb{Q}\left(\alpha_{13}\right)=\mathbb{Q}(\sqrt{-3 \sqrt{13}+1})$ is an imaginary quadratic extension of $\mathbb{Q}\left(\beta_{13}\right)$; so $\mathbb{Q}\left(\alpha_{13}\right)$ is a CM-field, and $\sigma$ acts as complex conjugation.

Let $f_{13}$ be the polynomial of degree 13 over $\mathbb{Q}\left(\alpha_{13}\right)[t]$ defined in Table 1 :

$$
\begin{aligned}
f_{13}(x)=\frac{1}{13} x^{13}+ & \left(\left(9 \beta_{13}-39\right) \alpha_{13}-6 \beta_{13}+24\right) t x^{11} \\
& +\left(\left(9 \beta_{13}-39\right) \alpha_{13}-12 \beta_{13}+51\right) t x^{10}+\cdots
\end{aligned}
$$

(note $f_{13}$ is the polynomial of $[5, \S 5.3]$ with $a_{1}=\alpha_{13}$ ). We have a nontrivial factorization $f_{13}\left(x_{1}\right)-f_{13}^{\sigma}\left(x_{2}\right)=A_{13}\left(x_{1}, x_{2}\right) B_{13}\left(x_{1}, x_{2}\right)$, where

$$
\begin{aligned}
A_{13}\left(x_{1}, x_{2}\right)= & x_{1}^{4}+x_{2}^{4}+\left(\beta_{13}-3\right) x_{1}^{2} x_{2}^{2}-9\left(3 \beta_{13}-14\right) t x_{1} x_{2}+12\left(47 \beta_{13}-202\right) t^{2} \\
& -\left(\left(\beta_{13}-4\right) \alpha_{13}+2\right) x_{1}^{3} x_{2}+\left(\left(\beta_{13}-4\right) \alpha_{13}-\beta_{13}+3\right) x_{1} x_{2}^{3} \\
& +3\left(\left(17 \beta_{13}-73\right) \alpha_{13}-12 \beta_{13}+50\right) t x_{1}^{2} \\
& -3\left(\left(17 \beta_{13}-73\right) \alpha_{13}-10 \beta_{13}+45\right) t x_{2}^{2} \\
& +3\left(\left(5 \beta_{13}-22\right) \alpha_{13}-9 \beta_{13}+38\right) t x_{1} \\
& -3\left(\left(5 \beta_{13}-22\right) \alpha_{13}+2 \beta_{13}-9\right) t x_{2} ;
\end{aligned}
$$

note that $A_{13}\left(x_{2}, x_{1}\right)=A_{13}\left(x_{1}, x_{2}\right)^{\sigma}$. Both $A_{13}$ and $B_{13}$ are absolutely irreducible. 
TABLE 1. Coefficients of the polynomial $f_{13}$ (from Example 4.5)

\begin{tabular}{|c|c|}
\hline$d$ & Coefficient of $x^{d}$ in $f_{13}$ \\
\hline 13 & $1 / 13$ \\
\hline 12 & 0 \\
\hline 11 & $\left(\left(9 \beta_{13}-39\right) \alpha_{13}-6 \beta_{13}+24\right) t$ \\
\hline 10 & $\left(\left(9 \beta_{13}-39\right) \alpha_{13}-12 \beta_{13}+51\right) t$ \\
\hline 9 & $\left(\left(-174 \beta_{13}+753\right) \alpha_{13}+\left(519 \beta_{13}-2217\right)\right) t^{2}$ \\
\hline 8 & $\left(\left(1620 \beta_{13}-6966\right) \alpha_{13}-36 \beta_{13}+162\right) t^{2}$ \\
\hline 7 & $\begin{array}{l}\left(\left(-29781 \beta_{13}+128115\right) \alpha_{13}+\left(11988 \beta_{13}-51651\right)\right) t^{3} \\
+\left(\left(1638 \beta_{13}-7047\right) \alpha_{13}-1305 \beta_{13}+5616\right) t^{2}\end{array}$ \\
\hline 6 & $\left(\left(-147933 \beta_{13}+636498\right) \alpha_{13}+\left(135999 \beta_{13}-585198\right)\right) t^{3}$ \\
\hline 5 & $\begin{array}{l}\left(\left(503631 \beta_{13}-2166939\right) \alpha_{13}-585387 \beta_{13}+2518938\right) t^{4} \\
+\left(\left(-18036 \beta_{13}+77598\right) \alpha_{13}+\left(119934 \beta_{13}-516051\right)\right) t^{3}\end{array}$ \\
\hline 4 & $\begin{array}{l}\left(\left(-1130922 \beta_{13}+4866156\right) \alpha_{13}-1672488 \beta_{13}+7196364\right) t^{4} \\
+\left(\left(71604 \beta_{13}-308097\right) \alpha_{13}-37719 \beta_{13}+162297\right) t^{3}\end{array}$ \\
\hline 3 & $\begin{array}{l}\left(\left(1827441 \beta_{13}-7863156\right) \alpha_{13}+\left(2618325 \beta_{13}-11266209\right)\right) t^{5} \\
+\left(\left(-8005635 \beta_{13}+34446465\right) \alpha_{13}+\left(3453192 \beta_{13}-14858316\right)\right) t^{4}\end{array}$ \\
\hline 2 & $\begin{array}{l}\left(\left(50157306 \beta_{13}-215815671\right) \alpha_{13}-31620618 \beta_{13}+136056429\right) t^{5} \\
+\left(\left(-3343518 \beta_{13}+14386410\right) \alpha_{13}+\left(3744792 \beta_{13}-16113006\right)\right) t^{4}\end{array}$ \\
\hline 1 & $\begin{array}{l}\left(\left(-27171504 \beta_{13}+116912916\right) \alpha_{13}+\left(11138796 \beta_{13}-47927700\right)\right) t^{6} \\
+\left(\left(73616121 \beta_{13}-316753659\right) \alpha_{13}-96852267 \beta_{13}+416733579\right) t^{5} \\
+\left(\left(770472 \beta_{13}-3315168\right) \alpha_{13}-303912 \beta_{13}+1307664\right) t^{4}\end{array}$ \\
\hline 0 & $\begin{array}{l}\left(\left(-48359916 \beta_{13}+208081872\right) \alpha_{13}-48359916 \beta_{13}\right) t^{6} \\
+\left(\left(-13260672 \beta_{13}+57057696\right) \alpha_{13}-13260672 \beta_{13}\right) t^{5}\end{array}$ \\
\hline
\end{tabular}

Example 4.6 (Polynomials of degree 15). Let $\alpha_{15}$ be an element of $\overline{\mathbb{Q}}$ satisfying

$$
\alpha_{15}^{2}-\alpha_{15}+4=0
$$

The involution $\sigma: \alpha_{15} \mapsto 4 / \alpha_{15}$ generates $\operatorname{Gal}\left(\mathbb{Q}\left(\alpha_{15}\right) / \mathbb{Q}\right)$. Observe that $\mathbb{Q}\left(\alpha_{15}\right)=$ $\mathbb{Q}(\sqrt{-15})$ is an imaginary quadratic field, and $\sigma$ acts as complex conjugation.

Let $f_{15}$ be the polynomial of degree 15 over $\mathbb{Q}\left(\alpha_{15}\right)[t]$ defined in Table 2 :

$$
f_{15}(x)=\frac{1}{15} x^{15}+\left(\alpha_{15}-1\right) t x^{13}+\left(\alpha_{15}+7\right) t x^{12}+\cdots
$$

(our $f_{15}$ is the polynomial of $[5, \S 5.4]$ with $a_{1}=\alpha_{15}$.) We have a nontrivial factorization $f_{15}\left(x_{1}\right)-\left(-f_{15}^{\sigma}\left(x_{2}\right)\right)=A_{15}\left(x_{1}, x_{2}\right) B_{15}\left(x_{1}, x_{2}\right)$, where

$$
\begin{aligned}
A_{15}\left(x_{1}, x_{2}\right)= & x_{1}^{7}-\left(\alpha_{15}-1\right) x_{1}^{6} x_{2}-2 x_{1}^{5} x_{2}^{2}+\left(7 \alpha_{15}-3\right) t x_{1}^{5}+\left(\alpha_{15}+1\right) x_{1}^{4} x_{2}^{3} \\
& +22 t x_{1}^{4} x_{2}+\left(5 \alpha_{15}+65\right) t x_{1}^{4}-\left(\alpha_{15}-2\right) x_{1}^{3} x_{2}^{4}-\left(10 \alpha_{15}+2\right) t x_{1}^{3} x_{2}^{2} \\
& -\left(50 \alpha_{15}-70\right) t x_{1}^{3} x_{2}+\left(9 \alpha_{15}-69\right) t^{2} x_{1}^{3}-2 x_{1}^{2} x_{2}^{5} \\
& +\left(10 \alpha_{15}-12\right) t x_{1}^{2} x_{2}^{3}-90 t x_{1}^{2} x_{2}^{2}+\left(39 \alpha_{15}+33\right) t^{2} x_{1}^{2} x_{2} \\
& +\left(210 \alpha_{15}-150\right) t^{2} x_{1}^{2}+\alpha_{15} x_{1} x_{2}^{6}+22 t x_{1} x_{2}^{4}+\left(50 \alpha_{15}+20\right) t x_{1} x_{2}^{3} \\
& -\left(39 \alpha_{15}-72\right) t^{2} x_{1} x_{2}^{2}+450 t^{2} x_{1} x_{2} \\
& -\left(\left(63 \alpha_{15}+45\right) t^{3}-\left(225 \alpha_{15}+900\right) t^{2}\right) x_{1}+x_{2}^{7}-\left(7 \alpha_{15}-4\right) t x_{2}^{5} \\
& -\left(5 \alpha_{15}-70\right) t x_{2}^{4}-\left(9 \alpha_{15}+60\right) t^{2} x_{2}^{3}-\left(210 \alpha_{15}-60\right) t^{2} x_{2}^{2} \\
& +\left(\left(63 \alpha_{15}-108\right) t^{3}-\left(225 \alpha_{15}-1125\right) t^{2}\right) x_{2}-675 t^{3} ;
\end{aligned}
$$

note that $A_{15}\left(x_{2}, x_{1}\right)=A_{15}\left(x_{1}, x_{2}\right)^{\sigma}$. Both $A_{15}$ and $B_{15}$ are absolutely irreducible.

Example 4.7 (Polynomials of degree 21). Let $\alpha_{21}$ be an element of $\overline{\mathbb{Q}}$ satisfying

$$
\alpha_{21}^{2}-\alpha_{21}+2=0 \text {. }
$$

The involution $\sigma: \alpha_{21} \mapsto 2 / \alpha_{21}$ generates $\operatorname{Gal}\left(\mathbb{Q}\left(\alpha_{21}\right) / \mathbb{Q}\right)$; note that $\mathbb{Q}\left(\alpha_{21}\right)=$ $\mathbb{Q}(\sqrt{-7})$ is an imaginary quadratic field, and $\sigma$ acts as complex conjugation. 
TABLE 2. Coefficients of the polynomial $f_{15}$ (from Example 4.6)

\begin{tabular}{|r|l|r|l|}
\hline$d$ & Coefficient of $x^{d}$ in $f_{15}(x)$ & $d$ & Coefficient of $x^{d}$ in $f_{15}(x)$ \\
\hline \hline 15 & $1 / 15$ & 11 & $-\left(5 \alpha_{15}+21\right) t^{2}$ \\
\hline 14 & 0 & 10 & $\left(74 \alpha_{15}-142\right) t^{2}$ \\
\hline 13 & $\left(\alpha_{15}-1\right) t$ & 9 & $-\frac{1}{3}\left(261 \alpha_{15}-349\right) t^{3}+\left(90 \alpha_{15}+240\right) t^{2}$ \\
\hline 12 & $\left(\alpha_{15}+7\right) t$ & 8 & $-\left(649 \alpha_{15}+703\right) t^{3}$ \\
\hline 7 & $\left(138 \alpha_{15}+717\right) t^{4}+\left(1380 \alpha_{15}-5760\right) t^{3}$ \\
\hline 6 & $-\left(2192 \alpha_{15}-7756\right) t^{4}+\left(2500 \alpha_{15}+2800\right) t^{3}$ \\
\hline 5 & $\frac{1}{5}\left(5835 \alpha_{15}-4743\right) t^{5}-\left(17790 \alpha_{15}-5400\right) t^{4}$ \\
\hline 4 & $\left(9699 \alpha_{15}+6153\right) t^{5}+\left(300 \alpha_{15}-74400\right) t^{4}$ \\
\hline 3 & $\left(243 \alpha_{15}-3591\right) t^{6}+\left(4680 \alpha_{15}+92880\right) t^{5}+\left(21375 \alpha_{15}+4500\right) t^{4}$ \\
\hline 2 & $\left(7254 \alpha_{15}-28062\right) t^{6}-\left(93600 \alpha_{15}-165600\right) t^{5}$ \\
\hline 1 & $-\left(945 \alpha_{15}+675\right) t^{7}+\left(52920 \alpha_{15}-48600\right) t^{6}-\left(54000 \alpha_{15}+216000\right) t^{5}$ \\
\hline 0 & $\left(675 \alpha_{15}-5400\right) t^{7}-\left(10800 \alpha_{15}-86400\right) t^{6}$ \\
\hline \hline
\end{tabular}

TABle 3. Coefficients of the polynomial $f_{21}$ (from Example 4.7)

\begin{tabular}{|r|l|r|l|}
\hline$d$ & Coefficient of $x^{d}$ in $f_{21}(x)$ & $d$ & Coefficient of $x^{d}$ in $f_{21}(x)$ \\
\hline \hline 21 & 1 & 20 & 0 \\
\hline 19 & $42 \alpha_{21}+42$ & 18 & $84 \alpha_{21}+84$ \\
\hline 17 & $2331 \alpha_{21}-861$ & 16 & $8820 \alpha_{21}-2604$ \\
\hline 15 & $46816 \alpha_{21}-64568$ & 14 & $227136 \alpha_{21}-306320$ \\
\hline 13 & $417060 \alpha_{21}-1450470$ & 12 & $1249248 \alpha_{21}-6783504$ \\
\hline 11 & $-1650124 \alpha_{21}-18355540$ & 10 & $-25341624 \alpha_{21}-54772872$ \\
\hline 9 & $-99408078 \alpha_{21}-104516426$ & 8 & $-414193752 \alpha_{21}-32069128$ \\
\hline 7 & $-1090995696 \alpha_{21}+266146344$ & 6 & $-2279293856 \alpha_{21}+2006258800$ \\
\hline 5 & $-4341402044 \alpha_{21}+5721876405$ & 4 & $-4332603072 \alpha_{21}+10737937392$ \\
\hline 3 & $-2459323342 \alpha_{21}+18242100282$ & 2 & $1708403396 \alpha_{21}+16523766868$ \\
\hline 1 & $8637088971 \alpha_{21}+9205492695$ & 0 & $4696767684 \alpha_{21}$ \\
\hline
\end{tabular}

Let $f_{21}$ be the polynomial of degree 21 over $k$ defined in Table 3 :

$$
f_{21}(x)=x^{21}+\left(42 \alpha_{21}+42\right) x^{19}+\left(84 \alpha_{21}+84\right) x^{18}+\cdots
$$

(note $f_{21}(x)=2^{21} g(x / 2)$, where $g$ is the polynomial of $[5, \S 5.5]$ with $a_{1}=\alpha_{21}$.) We have a nontrivial factorization $f_{21}\left(x_{1}\right)-f_{21}^{\sigma}\left(x_{2}\right)=A_{21}\left(x_{1}, x_{2}\right) B_{21}\left(x_{1}, x_{2}\right)$, where

$$
\begin{aligned}
A_{21}\left(x_{1}, x_{2}\right)= & x_{1}^{5}+\left(\alpha_{21}+1\right) x_{1}^{4} x_{2}+2 \alpha_{21} x_{1}^{3} x_{2}^{2}+\left(10 \alpha_{21}+18\right) x_{1}^{3} \\
& +\left(2 \alpha_{21}-2\right) x_{1}^{2} x_{2}^{3}+\left(32 \alpha_{21}-8\right) x_{1}^{2} x_{2}+\left(20 \alpha_{21}+4\right) x_{1}^{2} \\
& +\left(\alpha_{21}-2\right) x_{1} x_{2}^{4}+\left(32 \alpha_{21}-24\right) x_{1} x_{2}^{2}+\left(32 \alpha_{21}-16\right) x_{1} x_{2} \\
& +\left(107 \alpha_{21}+55\right) x_{1}-x_{2}^{5}+\left(10 \alpha_{21}-28\right) x_{2}^{3}+\left(20 \alpha_{21}-24\right) x_{2}^{2} \\
& +\left(107 \alpha_{21}-162\right) x_{2}+136 \alpha_{21}-68 .
\end{aligned}
$$

Note that $A_{21}\left(x_{1}, x_{2}\right)=-A_{21}^{\sigma}\left(x_{2}, x_{1}\right)$. Both $A_{21}$ and $B_{21}$ are absolutely irreducible.

Example 4.8 (Polynomials of degree 31). Let $\alpha_{31}$ and $\beta_{31}$ be elements of $\overline{\mathbb{Q}}$ satisfying

$$
\beta_{31}^{3}-13 \beta_{31}^{2}+46 \beta_{31}-32=0 \quad \text { and } \quad \alpha_{31}^{2}-1 / 2\left(\beta_{31}^{2}-7 \beta_{31}+4\right) \alpha_{31}+\beta_{31}=0 .
$$

The involution $\sigma: \alpha_{31} \mapsto \beta_{31} / \alpha_{31}$ generates $\operatorname{Gal}\left(\mathbb{Q}\left(\alpha_{31} / \mathbb{Q}\left(\beta_{31}\right)\right)\right.$; note that $\mathbb{Q}\left(\beta_{31}\right)$ is a totally real cubic field, and $\mathbb{Q}\left(\alpha_{31}\right)$ is a totally imaginary quadratic extension of $\mathbb{Q}\left(\beta_{31}\right)$; so $\mathbb{Q}\left(\alpha_{31}\right)$ is a CM-field, and $\sigma$ acts as complex conjugation. 
TABLE 4. Coefficients of the polynomial $f_{31}$ (from Example 4.8): degrees 14 through 31

\begin{tabular}{|c|c|}
\hline$d$ & Coefficient of $x^{d}$ in $f_{31}(x)$ \\
\hline ב31 & $1 / 1 / 31$ \\
\hline 30 & 0 \\
\hline 29 & $\frac{-1}{4}\left(\beta_{31}^{2}-5 \beta_{31}-10\right) \alpha_{31}+\beta_{31}^{2}-7 \beta_{31}+12$ \\
\hline 28 & $\frac{-1}{2}\left(\beta_{31}^{2}-5 \beta_{31}-10\right) \alpha_{31}+2 \beta_{31}^{2}-14 \beta_{31}+24$ \\
\hline 27 & $\frac{1}{4}\left(43 \beta_{31}^{2}-1011 \beta_{31}+2854\right) \alpha_{31}+\frac{1}{2}\left(453 \beta_{31}^{2}-3055 \beta_{31}+3248\right)$ \\
\hline 26 & $\left(41 \beta_{31}^{2}-977 \beta_{31}+2802\right) \alpha_{31}+886 \beta_{31}^{2}-5986 \beta_{31}+6496$ \\
\hline 25 & $\frac{-1}{4}\left(17521 \beta_{31}^{2}-74509 \beta_{31}-60450\right) \alpha_{31}+14092 \beta_{31}^{2}-77272 \beta_{31}+68380$ \\
\hline 24 & $\frac{-1}{2}\left(48519 \beta_{31}^{2}-204491 \beta_{31}-184718\right) \alpha_{31}+80184 \beta_{31}^{2}-442624 \beta_{31}+403208$ \\
\hline 23 & $\frac{-1}{4}\left(1776161 \beta_{31}^{2}-9373621 \beta_{31}+3292454\right) \alpha_{31}+\frac{1}{2}\left(2041603 \beta_{31}^{2}-11554557 \beta_{31}+8612300\right)$ \\
\hline 22 & $-\left(2942318 \beta_{31}^{2}-15455046 \beta_{31}+5475220\right) \alpha_{31}+7037348 \beta_{31}^{2}-40203740 \beta_{31}+30052880$ \\
\hline 21 & $\frac{-1}{4}\left(109481293 \beta_{31}^{2}-596329857 \beta_{31}+368885054\right) \alpha_{31}+46576255 \beta_{31}^{2}-265263537 \beta_{31}+187276364$ \\
\hline 20 & $\begin{array}{l}\frac{-1}{2}\left(384855193 \beta_{31}^{2}-2112196605 \beta_{31}+1408837958\right) \alpha_{31} \\
+307371526 \beta_{31}^{2}-1742220634 \beta_{31}+1208790968\end{array}$ \\
\hline 19 & $\begin{array}{l}\frac{-1}{4}\left(5290184805 \beta_{31}^{2}-29820077413 \beta_{31}+21851209042\right) \alpha_{31} \\
+\frac{1}{2}\left(2521588153 \beta_{31}^{2}-13978683691 \beta_{31}+9274523664\right)\end{array}$ \\
\hline 18 & $\begin{array}{l}-\left(8697236749 \beta_{31}^{2}-49763738685 \beta_{31}+38332116082\right) \alpha_{31} \\
+5911141274 \beta_{31}^{2}-32035079054 \beta_{31}+20126371040\end{array}$ \\
\hline 17 & $\begin{array}{l}\frac{-1}{4}\left(186111470445 \beta_{31}^{2}-1067698578649 \beta_{31}+833400031142\right) \alpha_{31} \\
+9484781350 \beta_{31}^{2}-47546236774 \beta_{31}+16736919932\end{array}$ \\
\hline 16 & $\begin{array}{l}\frac{-1}{2}\left(494148938071 \beta_{31}^{2}-2839948380571 \beta_{31}+2256232777618\right) \alpha_{31} \\
-61154690060 \beta_{31}^{2}+368281842924 \beta_{31}-366207873944\end{array}$ \\
\hline 15 & $\begin{array}{l}\frac{-1}{2}\left(2214031635615 \beta_{31}^{2}-12716268790027 \beta_{31}+10156041792602\right) \alpha_{31} \\
-960101407852 \beta_{31}^{2}+5535136704359 \beta_{31}-4581193619353\end{array}$ \\
\hline 14 & $\begin{array}{l}-\left(4484463959192 \beta_{31}^{2}-25746958551032 \beta_{31}+20641481233168\right) \alpha_{31} \\
-8423937387072 \beta_{31}^{2}+48228838157776 \beta_{31}-38143305780784\end{array}$ \\
\hline
\end{tabular}

Let $f_{31}$ be the polynomial of degree 31 over $k$ defined in Tables 4 and 5 :

$$
\begin{aligned}
f_{31}(x)= & \frac{1}{31} x^{31}-\left(\frac{1}{4}\left(\beta_{31}^{2}-5 \beta_{31}-10\right) \alpha_{31}-\left(\beta_{31}^{2}-7 \beta_{31}+12\right)\right) x^{29} \\
& -\left(\frac{1}{2}\left(\beta_{31}^{2}-5 \beta_{31}-10\right) \alpha_{31}-\left(2 \beta_{31}^{2}-14 \beta_{31}+24\right)\right) x^{28}+\cdots
\end{aligned}
$$

(note $f_{31}(x)=2^{31} g(x / 2) / 31$, where $g$ is the polynomial of $[5, \S 5.6]$ with $a_{1}=\alpha_{31}$ ). We have a nontrivial factorization $f_{31}\left(x_{1}\right)-f_{31}^{\sigma}\left(x_{2}\right)=A_{31}\left(x_{1}, x_{2}\right) B_{31}\left(x_{1}, x_{2}\right)$, where

$$
\begin{aligned}
A_{31}\left(x_{1}, x_{2}\right)= & x_{1}^{15}+\left(\frac{1}{4}\left(\beta_{31}^{2}-9 \beta_{31}+14\right) \alpha_{31}-\beta_{31}+4\right) x_{1}^{14} x_{2} \\
& +\cdots \\
& +\left(\frac{1}{4}\left(\beta_{31}^{2}-9 \beta_{31}+14\right) \alpha_{31}+\frac{1}{2}\left(\beta_{31}^{2}-7 \beta_{31}+2\right)\right) x_{1} x_{2}^{14}-x_{2}^{15}
\end{aligned}
$$

is a polynomial of total degree 15 satisfying $A_{31}\left(x_{1}, x_{2}\right)=-A_{31}^{\sigma}\left(x_{2}, x_{1}\right)$. Both $A_{31}$ and $B_{31}$ are absolutely irreducible.

If $(f, g)$ and $\left(f^{\prime}, g^{\prime}\right)$ are equivalent pairs of polynomials, and $(\mathcal{X}, \mathcal{Y})$ and $\left(\mathcal{X}^{\prime}, \mathcal{Y}^{\prime}\right)$ the families of pairs of curves associated to $(f, g)$ and $\left(f^{\prime}, g^{\prime}\right)$ by the linear or quadratic constructions of $\S 2$, then $(\mathcal{X}, \mathcal{Y})$ and $\left(\mathcal{X}^{\prime}, \mathcal{Y}^{\prime}\right)$ are isomorphic. Indeed, suppose $f^{\prime}(x)=c f\left(a_{1} x+b_{1}\right)+d$ and $g^{\prime}(x)=c g\left(a_{2} x+b_{2}\right)+d$ for some $a_{1}, b_{1}, a_{2}$, $b_{2}, c$, and $d$ in $k$ with $c, a_{1}$ and $a_{2}$ nonzero. The isomorphism $(\mathcal{X}, \mathcal{Y}) \rightarrow\left(\mathcal{X}^{\prime}, \mathcal{Y}^{\prime}\right)$ is defined by $\left(x_{i}, y_{i}\right) \mapsto\left(a_{i} x_{1}+b_{i}, c^{1 / 2} y_{i}\right)$ and $s \mapsto(s+d) / c$ for the linear construction, and by $\left(x_{i}, y_{i}\right) \mapsto\left(a_{i} x_{1}+b_{i}, c y_{i}\right)$ and $\left(s_{1}, s_{2}\right) \mapsto\left(\left(s_{2}+2 d\right) / c,\left(s_{2}+d s_{1}+d^{2}\right) / c^{2}\right)$ for the quadratic construction.

Lemma 4.2. With the notation of Examples 4.3 through 4.8: 
TABle 5. Coefficients of the polynomial $f_{31}$ (from Example 4.8): degrees 13 through 0

\begin{tabular}{|c|c|}
\hline$d$ & Coefficient of $x^{d}$ in $f_{31}(x)$ \\
\hline 13 & $\begin{array}{l}\frac{-1}{4}\left(63813876335979 \beta_{31}^{2}-367007052549207 \beta_{31}+296370094708306\right) \alpha_{31} \\
-52401417590341 \beta_{31}^{2}+299616088960507 \beta_{31}-233801230247956\end{array}$ \\
\hline 12 & $\begin{array}{l}\frac{-1}{2}\left(84595067837587 \beta_{31}^{2}-488413358269471 \beta_{31}+399412816680130\right) \alpha_{31} \\
-289909376875898 \beta_{31}^{2}+1656226239390086 \beta_{31}-1283082623470440\end{array}$ \\
\hline 11 & $\begin{array}{l}\frac{-1}{4}\left(276978123366339 \beta_{31}^{2}-1621224937178539 \beta_{31}+1399602523915382\right) \alpha_{31} \\
-\frac{1}{2}\left(2756444217062133 \beta_{31}^{2}-15735604159262247 \beta_{31}+12145338716741672\right)\end{array}$ \\
\hline 10 & $\begin{array}{l}\left(164996225556971 \beta_{31}^{2}-911562557305603 \beta_{31}+591654846604694\right) \alpha_{31} \\
-5775442801086222 \beta_{31}^{2}+32951684149353882 \beta_{31}-25388487691873328\end{array}$ \\
\hline 9 & $\begin{array}{l}\frac{1}{4}\left(8153525016709589 \beta_{31}^{2}-46226784686942241 \beta_{31}+34465661136373590\right) \alpha_{31} \\
-21765717548444108 \beta_{31}^{2}+124141863300896800 \beta_{31}-95543137393851316\end{array}$ \\
\hline 8 & $\begin{array}{l}\frac{1}{2}\left(21507787300535771 \beta_{31}^{2}-122360462847124879 \beta_{31}+92829028744745354\right) \alpha_{31} \\
-71879278651985336 \beta_{31}^{2}+409920655394903344 \beta_{31}-315310665232998936 \\
\end{array}$ \\
\hline 7 & $\begin{array}{l}\frac{1}{4}\left(172549107727779319 \beta_{31}^{2}-982848727924637571 \beta_{31}+750639722104375338\right) \alpha_{31} \\
-\frac{1}{2}\left(418768591310359209 \beta_{31}^{2}-2388174561757656643 \beta_{31}+1836495177429186664\right)\end{array}$ \\
\hline 6 & $\begin{array}{l}\left(138365490236826262 \beta_{31}^{2}-788531695474992526 \beta_{31}+604055823258954628\right) \alpha_{31} \\
-530716158860110596 \beta_{31}^{2}+3026599060976364972 \beta_{31}-2327045484274854432\end{array}$ \\
\hline 5 & $\begin{array}{l}\frac{1}{4}\left(1461494193805567097 \beta_{31}^{2}-8330939217188411741 \beta_{31}+6391346186593069190\right) \alpha_{31} \\
-1132691540565214443 \beta_{31}^{2}+6459518768862357533 \beta_{31}-4965998974814592772\end{array}$ \\
\hline 4 & $\begin{array}{l}\frac{1}{2}\left(1590470411372385357 \beta_{31}^{2}-9067705413825934465 \beta_{31}+6962808016837221182\right) \alpha_{31} \\
-1998830101622128910 \beta_{31}^{2}+11398708269008017730 \beta_{31}-8762745131128427944\end{array}$ \\
\hline 3 & $\begin{array}{l}\frac{1}{4}\left(5458654735992646373 \beta_{31}^{2}-31124897594589327589 \beta_{31}+23912314632422881618\right) \alpha_{31} \\
+\frac{1}{2}\left(-5512701081507844017 \beta_{31}^{2}+31436273506520022779 \beta_{31}-24164866978481400776\right)\end{array}$ \\
\hline 2 & $\begin{array}{l}\left(1756872157897042025 \beta_{31}^{2}-10018233805014343961 \beta_{31}+7698964739179717386\right) \alpha_{31} \\
-2631501460411936866 \beta_{31}^{2}+15005661005014590390 \beta_{31}-11533152751494298576 \\
\end{array}$ \\
\hline 1 & $\begin{array}{l}\frac{1}{4}\left(6099047880687359369 \beta_{31}^{2}-34780055276291665989 \beta_{31}+26734049819113493038\right) \alpha_{31} \\
-1489705167473733478 \beta_{31}^{2}+8494536217258921566 \beta_{31}-6527531886543984036\end{array}$ \\
\hline 0 & $\begin{array}{l}\frac{1}{2}\left(1290343630884751523 \beta_{31}^{2}-7358426308111535607 \beta_{31}+5657092118674073402\right) \alpha_{31} \\
-2127333184925614050 \beta_{31}+1673979108081725054\end{array}$ \\
\hline
\end{tabular}

(1) For $n=11,21$, 31, the image of the family $\mathcal{X}: y^{2}=f_{n}(x)+s$ in $\mathcal{H}_{(n-1) / 2}$ is one-dimensional;

(2) For $n=7$, 13, 15, the image of the family $\mathcal{X}: y^{2}=f_{n}(x)+s$ in $\mathcal{H}_{(n-1) / 2}$ is two-dimensional;

(3) For $n=11,21$, 31, the image of the family $\mathcal{X}: y^{2}=f_{n}(x)^{2}+s_{1} f_{n}(x)+s_{2}$ in $\mathcal{H}_{(n-1)}$ is two-dimensional;

(4) For $n=7,13,15$, the image of the family $\mathcal{X}: y^{2}=f_{n}(x)^{2}+s_{1} f_{n}(x)+s_{2}$ in $\mathcal{H}_{(n-1)}$ is three-dimensional.

Proof. We will show that only finitely many curves in each family $\mathcal{X}$ can be isomorphic to a given element of $\mathcal{X}$. This implies that intersection of $\mathcal{X}(\overline{\mathbb{Q}})$ with the isomorphism class of a curve $X$ in $\mathcal{X}(\overline{\mathbb{Q}})$ is finite, and hence that the map from $\mathcal{X}(\overline{\mathbb{Q}})$ into the moduli space of hyperelliptic curves is finite. The dimension of the image of $\mathcal{X}$ in the moduli space is then equal to the number of parameters of $\mathcal{X}$.

Consider (1): if $X: y^{2}=c_{0} x^{n}+c_{1} x^{n-1}+c_{2} x^{n-2}+\cdots+c_{n}$ is a hyperelliptic curve in the family $\mathcal{X}$, then the coefficients $c_{i}$ satisfy conditions

$$
(\mathrm{A}): c_{0}=1, \quad(\mathrm{~B}): c_{1}=0, \quad(\mathrm{C}): c_{2} \neq 0, \quad \text { and } \quad(\mathrm{D}): c_{3}=\kappa_{n} c_{2},
$$

where $\kappa_{11}=2 / \alpha_{11}, \kappa_{21}=2$, and $\kappa_{31}=2$. 
If $X^{\prime}:\left(y^{\prime}\right)^{2}=f_{n}\left(x^{\prime}\right)+s^{\prime}$ is isomorphic to $X$, then there exists a birational map $\psi: X \rightarrow X^{\prime}$ defined by

$$
\psi:(x, y) \mapsto\left(x^{\prime}, y^{\prime}\right)=\left(\frac{\alpha x+\beta}{\gamma x+\delta}, \frac{\epsilon y}{(\gamma x+\delta)^{(n+1) / 2}}\right)
$$

with $\alpha, \beta, \gamma, \delta$, and $\epsilon$ in $\overline{\mathbb{Q}}$ sastisfying $\epsilon \neq 0$ and $\alpha \delta-\beta \gamma \neq 0$, and $X^{\prime}$ has a defining equation $X^{\prime}: y^{2}=\epsilon^{-2}(\gamma x+\delta)^{n+1}\left(f_{n}((\alpha x+\beta) /(x+\delta))+s\right)$.

If $\gamma=0$, then we may take $\delta=1$, so $\psi(x, y)=(\alpha x+\beta, \epsilon y)$. If $X^{\prime}$ is in $\mathcal{X}$ then it satisfies (A), (B), (C), and (D). Condition (A) implies $\alpha^{n}=\epsilon^{2}$, while (B) forces $\beta=0$. The coefficients of $x^{n-2}$ and $x^{n-3}$ in $f_{n}(\alpha x)+s$ are then $\alpha^{n-2} c_{2}$ and $\alpha^{n-3} c_{3}=\alpha^{n-3} \kappa_{n} c_{2}$, whereupon (C) and (D) imply $\alpha=1$, and hence $\epsilon= \pm 1$. We conclude that $\psi$ must be either the identity map or the hyperelliptic involution, depending on the sign of $\epsilon$; in either case, $X^{\prime}=X$.

If $\gamma \neq 0$, then we may take $\gamma=1$. For the hyperelliptic polynomial of $X^{\prime}$ to have degree $n$ we must have $\delta=-\rho$, where $\rho$ is one of the roots of $f_{n}(x)+s$. Conditions $(\mathrm{A}),(\mathrm{B}),(\mathrm{C})$, and (D) then uniquely determine $\alpha, \beta, \gamma$, and $\epsilon$ (up to sign) in terms of $\rho$ and $\kappa_{n}$. Since there were only $n$ possible choices of $\rho$, we find that there are only $2 n$ possible choices for $\psi$, and only $n$ modulo the hyperelliptic involution.

We have shown that there are only $n+1$ possible defining equations for curves in $\mathcal{X}$ isomorphic to $X$ (in fact, each corresponds to a choice of Weierstrass point of $X$ ). has a unique defining equation (since the coefficient of $x^{0}$ in the defining equation uniquely determines a point of the parameter space); hence there are at most $n+1$ curves in $\mathcal{X}$ isomorphic to $X$.

The proof is identical for (2), though in this case we must restrict to the open subfamily of $\mathcal{X}$ where $t \neq 0$ (so that (B) holds), and take $\kappa_{7}=1, \kappa_{13}=-\left(\left(2 \beta_{13}-\right.\right.$ 9) $\left.\alpha_{6}-\beta_{13}+3\right) / 3$, and $\kappa_{15}=-2 \alpha_{15}+1$. Again, each curve in $\mathcal{X}$ has a unique defining equation: the coefficients of $x^{0}$ and $x^{n}$ uniquely determine a point of the parameter space.

The proof for (3) and (4) is similar, and we only sketch it here. This time the curves $X: y^{2}=\sum_{i=0}^{2 n} c_{i} x^{2 n-i}$ in $\mathcal{X}$ (or the subfamily where $t=0$ in (4)) satisfy

$$
\begin{array}{ll}
\left(\mathrm{A}^{\prime}\right): c_{0}=1, & \left(\mathrm{~B}^{\prime}\right): c_{1}=0, \quad\left(\mathrm{C}^{\prime}\right): c_{2} \neq 0, \\
\left(\mathrm{D}^{\prime}\right): c_{3}=\kappa_{n} c_{2}, & \left(\mathrm{E}^{\prime}\right): c_{4} \neq 0, \quad\left(\mathrm{~F}^{\prime}\right): c_{5}=\lambda_{n} c_{4},
\end{array}
$$

with $\kappa_{n}$ defined as above and

$$
\begin{aligned}
\lambda_{7}= & \frac{1}{277}\left(44 \alpha_{7}+502\right), \\
\lambda_{11}= & \frac{-1}{1049}\left(1444 \alpha_{11}+1292\right), \\
\lambda_{13}= & \left.\frac{-1}{24470889}\left(32177912 \beta_{13}-144562170\right) \alpha_{13}-14922610 \beta_{13}+44742102\right), \\
\lambda_{15}= & \frac{-1}{3061}\left(11624 \alpha_{15}-8242\right), \\
\lambda_{21}= & \frac{-1}{24889}\left(1872 \alpha_{2} 121-98252\right), \text { and } \\
\lambda_{31}= & \frac{1}{5572804315201}\left(\left(-23763234474 \beta_{31}^{2}+308913876190 \beta_{31}-904140145396\right) \alpha_{31}\right. \\
& \left.+\left(45939160324 \beta_{31}^{2}-413033009792 \beta_{31}+22556391264028\right)\right) .
\end{aligned}
$$

As before, the defining equation of any curve in $\mathcal{X}$ isomorphic to $X$ is uniquely determined by $\left(\mathrm{A}^{\prime}\right),\left(\mathrm{B}^{\prime}\right),\left(\mathrm{C}^{\prime}\right),\left(\mathrm{D}^{\prime}\right),\left(\mathrm{E}^{\prime}\right),\left(\mathrm{F}^{\prime}\right)$, and the choice of a root of $f_{n}(x)^{2}+$ $s_{1} f_{n}(x)+s_{2}$. The curves in $\mathcal{X}$ have unique defining equations, since the coefficients of $x^{0}$ and $x^{n}$ (and $x^{2 n-2}$ in (4)) uniquely determine the corresponding point of the parameter space. Hence there are at most $2 n$ curves in $\mathcal{X}$ isomorphic to $X$.

\section{Explicit Complex And Real Multiplications}

We now apply the methods of $\S 2$ and $\S 3$ to the factorizations in Examples 4.1 and 4.2. Most of the resulting families have already been investigated elsewhere, so we treat them only briefly here. Throughout this section $n$ denotes an odd prime. 
Example 5.1. The linear construction on $\left(x^{n}, x^{n}\right)$ yields a family $(\mathcal{X}, \mathcal{X})$ of pairs of hyperelliptic curves of genus $(n-1) / 2$, defined by $\mathcal{X}: y_{i}^{2}=x_{i}^{n}+s$. The curves in $\mathcal{X}$ are all isomomorphic to the curve $X: y_{i}^{2}=x_{i}^{n}+1\left(\right.$ via $\left.\left(x_{i}, y_{i}\right) \mapsto\left(\sqrt[n]{s} x_{i}, \sqrt{s} y_{i}\right)\right)$. The Jacobian $J_{X}$ is absolutely simple by [23, Example 8.4.(1)]. The correspondence $C=V\left(y_{1}-y_{2}, x_{1}-\zeta_{n}^{e} x_{2}\right)$ on $X \times X$ induces an endomorphism $\phi$ of $J_{X}$. Clearly $d\left(x_{1}^{i}\right) / y_{1}=\zeta_{n}^{i e} d\left(x_{2}^{i}\right) / y_{2}$ on $C$, so

$$
D_{X, Y}(\phi)=\operatorname{diag}\left(\zeta_{n}^{e}, \zeta_{n}^{2 e}, \ldots, \zeta_{n}^{(n-1) e / 2}\right) .
$$

The factors $x_{1}-\zeta_{n}^{e} x_{2}$ of $x_{1}^{n}-x_{2}^{n}$ therefore correspond to explicit generators for a subring of $\operatorname{End}\left(J_{X}\right)$ isomorphic to $\mathbb{Z}\left[\zeta_{n}\right]$.

Example 5.2. The quadratic construction on $\left(x^{n}, x^{n}\right)$ yields a two-parameter family $\left(\mathcal{X}: y_{1}^{2}=x_{1}^{2 n}+s_{1} x_{1}^{n}+s_{2}, \mathcal{X}: y_{2}^{2}=x_{2}^{2 n}+s_{1} x_{2}^{n}+s_{2}\right)$ of pairs of hyperelliptic curves of genus $n-1$. Twisting by $\left(x_{i}, y_{i}\right) \mapsto\left(s_{2}^{1 / 2 n} x_{i}, s_{2}^{1 / 2} y_{i}\right)$, we reduce to the oneparameter family $\mathcal{X}^{\prime}: y^{2}=x^{2 n}+s_{1} x^{n}+1$ of [26, Remark after Proposition 3]. The family $\mathcal{X}^{\prime}$ has an involution $\iota:(x, y) \mapsto\left(1 / x, y / x^{n}\right)$ which is clearly not the hyperelliptic involution, so $\mathcal{J}_{\mathcal{X}^{\prime}}$ is reducible. The correspondences $V\left(y_{1}-y_{2}, x_{2}-\zeta_{n}^{i} x_{1}\right)$ on $\mathcal{X}^{\prime} \times_{T} \mathcal{X}^{\prime}$ induce endomorphisms generating a subring of $\operatorname{End}\left(\mathcal{J}_{\mathcal{X}^{\prime}}\right)$ isomorphic to $\mathbb{Z}\left[\zeta_{n}\right]$, as in Example 5.1. The quotient of $\mathcal{X}^{\prime}$ by $\langle\iota\rangle$ is a one-parameter family of curves of genus $(n-1) / 2$ whose Jacobians have Real Multiplication by $\mathbb{Z}\left[\zeta_{n}+\zeta_{n}^{-1}\right]$.

Example 5.3. Let $D_{n}$ and $A_{n, i}$ be defined as in Example 4.2. The linear construction on $\left(D_{n}(x), D_{n}(x)\right)$ yields a one-parameter family

$$
\left(\mathcal{X}: y_{1}^{2}=D_{n}\left(x_{1}\right)+s, \mathcal{X}: y_{2}^{2}=D_{n}\left(x_{2}\right)+s\right)
$$

of pairs of hyperelliptic curves of genus $(n-1) / 2$ over $\mathbb{Q}$. The family $\mathcal{X}$ is identical to the family $\mathcal{C}_{t}$ of [26, Theorem 1]. It is shown in [26] that the endomorphisms induced by $V\left(y_{1}-y_{2}, A_{n, i}\left(x_{1}, x_{2}\right)\right)$ for $1 \leq i \leq(n-1) / 2$ generate a subring of $\operatorname{End}\left(\mathcal{J}_{\mathcal{X}}\right)$ isomorphic to $\mathbb{Z}\left[\zeta_{n}+\zeta_{n}^{-1}\right]$ (while $V\left(y_{1}-y_{2}, x_{1}-x_{2}\right)$ clearly induces $\left.[1]_{\mathcal{J}_{\mathcal{X}}}\right)$. It is shown that $\mathcal{J}_{\mathcal{X}}$ is absolutely simple for $n>5$ in [26, Corollary 6] and for $n=5$ in [17, Remark 15]. The cases $n=5$ and $n=7$ of this construction appear as families of efficiently computable endomorphisms in [17].

Example 5.4. Applied to $\left(D_{n}(x), D_{n}(x)\right)$, the quadratic construction yields a twoparameter family

$$
\left(\mathcal{X}: y_{1}^{2}=D_{n}\left(x_{1}\right)^{2}+s_{1} D_{n}\left(x_{1}\right)+s_{2}, \mathcal{X}: y_{2}^{2}=D_{n}\left(x_{2}\right)^{2}+s_{1} D_{n}\left(x_{2}\right)+s_{2}\right)
$$

of pairs of hyperelliptic curves of genus $n-1$. We have a nontrivial factorization

$$
\begin{aligned}
& \left(D_{n}\left(x_{1}\right)^{2}+s_{1} D_{n}\left(x_{1}\right)\right)-\left(D_{n}\left(x_{2}\right)^{2}+s_{1} D_{n}\left(x_{2}\right)\right. \\
= & \left(D_{n}\left(x_{1}\right)-D_{n}\left(x_{2}\right)\right)\left(D_{n}\left(x_{1}\right)+D_{n}\left(x_{2}\right)+s_{1}\right) \\
= & \left(\left(x_{1}-x_{2}\right) \prod_{i=1}^{(n-1) / 2} A_{n, i}\left(x_{1}, x_{2}\right)\right)\left(\left(D_{n}\left(x_{1}\right)+D_{n}\left(x_{2}\right)+s_{1}\right)\right) .
\end{aligned}
$$

The correspondences $V\left(y_{1}-y_{2}, A_{n, i}\left(x_{1}, x_{2}\right)\right)$ on $X \times_{T} Y$ induce endomorphisms $\phi_{i}$ of $\mathcal{J}_{\mathcal{X}}$ for $1 \leq i \leq(n-1) / 2$; the diagonal correspondence $V\left(y_{1}-y_{2}, x_{1}-x_{2}\right)$ induces $[1]_{J_{X}}$. The matrix $D_{\mathcal{X}, \mathcal{X}}\left(\phi_{i}\right)$ is an endomorphism of $\Omega(X)$. Since $D_{\mathcal{X}, \mathcal{X}}\left(\phi_{i}\right)$ is lower-triangular, its characteristic polynomial (and hence that of $\phi_{i}$ ) is

$$
P(x)=\prod_{j=1}^{(n-1)}\left(x-t_{j, j}\right),
$$

where $t_{j, j}$ is the $j^{\text {th }}$ entry on the diagonal of $D_{\mathcal{X}, \mathcal{X}}\left(\phi_{i}\right)$ : that is, $t_{j, j}$ is the leading coefficient of the trace $t_{j}=\operatorname{Tr}_{\Omega(X)}^{\Omega\left(C_{i}\right)}\left(d\left(x_{1}^{j}\right) / y_{1}\right)$ written as a polynomial in $x_{2}$. We have

$$
A_{n, i}\left(x_{1}, x_{2}\right)=x_{1}^{2}-\left(\zeta_{n}^{i}+\zeta_{n}^{-i}\right) x_{2} \cdot x_{1}+\left(x_{2}^{2}+\zeta_{n}^{i}-\zeta_{n}^{-i}\right)
$$


$\mathrm{SO}$

$$
\begin{aligned}
& t_{1}=\left(\zeta_{n}^{i}+\zeta_{n}^{-i}\right) x_{2}, \\
& t_{2}=\left(\zeta_{n}^{2 i}+\zeta_{n}^{-2 i}\right) x_{2}^{2}-2\left(\zeta_{n}^{i}-\zeta_{n}^{-i}\right), \text { and } \\
& t_{j}=\left(\zeta_{n}^{i}+\zeta_{n}^{-i}\right) x_{2} t_{j-1}-\left(x_{2}^{2}+\zeta_{n}^{i}-\zeta_{n}^{-i}\right) t_{j-2} \text { for } j>2 ;
\end{aligned}
$$

in particular, the coefficients $t_{j, j}$ satisfy $t_{1,1}=\zeta_{n}^{i}+\zeta_{n}^{-i}, t_{2,2}=\zeta_{n}^{2 i}+\zeta_{n}^{-2 i}$, and

$$
t_{j, j}=\left(\zeta_{n}^{i}+\zeta_{n}^{-i}\right) t_{j-1, j-1}-t_{j-2, j-2} \quad \text { for } j>2 .
$$

Solving the second-order linear recurrence, we find $t_{j, j}=\zeta_{n}^{i j}+\zeta_{n}^{-i j}$ for all $j>0$, so

$$
P(x)=\prod_{j=1}^{(n-1)}\left(x-\left(\zeta_{n}^{i j}+\zeta_{n}^{-i j}\right)\right)=m(x)^{2},
$$

where $m$ is the minimal polynomial of $\zeta_{n}+\zeta_{n}^{-1}$ over $\mathbb{Q}$; hence $m\left(\phi_{i}\right)=0$. We conclude that $\phi_{i}$ generates an explicit subring of $\operatorname{End}\left(\mathcal{J}_{\mathcal{X}}\right)$ isomorphic to $\mathbb{Z}\left[\zeta_{n}+\zeta_{n}^{-1}\right]$.

\section{FAMilies of EXPLiCit isogenies}

We now apply the methods of $\S 2$ and $\S 3$ to the factorizations in Examples 4.3 through 4.8. The examples in this section form the proof of Theorem 1.1.

Example 6.1. Let $f_{7}, A_{7}, \alpha_{7}$, and $\sigma$ be as in Example 4.3. The linear construction on $\left(f_{7}, f_{7}^{\sigma}\right)$ yields a two-parameter family

$$
\left(\mathcal{X}: y_{1}^{2}=f_{7}\left(x_{1}\right)+s, \mathcal{Y}: y_{2}^{2}=f_{7}^{\sigma}\left(x_{2}\right)+s\right)
$$

of pairs of hyperelliptic curves of genus 3 defined over $\mathbb{Q}\left(\alpha_{7}\right)$. Specializing $\mathcal{X}$ at $(s, t)=(1,0)$ we obtain the curve $X$ of Example 5.1 with $n=7$, where we noted that $J_{X}$ was absolutely simple; hence the generic fibre of $\mathcal{J}_{\mathcal{X}}$ is absolutely simple.

The correspondence $V\left(y_{1}-y_{2}, A_{7}\left(x_{1}, x_{2}\right)\right)$ on $\mathcal{X} \times_{T} \mathcal{Y}$ induces a homomorphism $\phi: \mathcal{J}_{\mathcal{X}} \rightarrow \mathcal{J}_{\mathcal{Y}}$. We have

and therefore

$$
D_{X, Y}(\phi)=\left(\begin{array}{ccc}
\alpha_{7} & 0 & 0 \\
0 & \alpha_{7} & 0 \\
\left(\alpha_{7}^{\sigma}-\alpha_{7}\right) t & 0 & \alpha_{7}^{\sigma}
\end{array}\right),
$$

$$
D_{X, Y}(\phi) D_{Y, X}\left(\phi^{\dagger}\right)=D_{X, Y}(\phi) D_{X, Y}(\phi)^{\sigma}=2 I_{3}
$$

so $\phi^{\dagger} \circ \phi=[2]_{\mathcal{X}}$; hence $\operatorname{ker} \phi \cong(\mathbb{Z} / 2 \mathbb{Z})^{3}$ by Lemma 3.2. The image of $\mathcal{J}_{\mathcal{X}}$ in $\mathcal{A}_{3}$ is two-dimensional by Lemma 4.2 and Torelli's theorem. We conclude that $\phi$ is a two-dimensional family of $(\mathbb{Z} / 2 \mathbb{Z})^{3}$-isogenies of (generically) absolutely simple Jacobians, thus proving Theorem 1.1 for the first row of the table.

Remark 6.1. More generally, given a hyperelliptic curve $X$ of genus 3 and a maximal 2-Weil isotropic subgroup $S$ of $J_{X}$ [2], there exists a (possibly reducible) curve $Y$ of genus 3 and a $(\mathbb{Z} / 2 \mathbb{Z})^{3}$-isogeny $\phi: J_{X} \rightarrow J_{Y}$ with kernel $S$ (both $Y$ and $\phi$ may be defined over a quadratic extension of the field of definition of $S$ ). In general, the curve $Y$ is not hyperelliptic. An algorithm which computes equations for $Y$ and $\phi$ in the case where $S$ is generated by differences of Weierstrass points appears in [25] (it is possible to show, using techniques similar to those of Lemma 3.4, that the kernel of the isogeny of Example 6.1 is not such a subgroup). The case where $X$ is non-hyperelliptic is treated in [19].

Example 6.2. Let $f_{7}, A_{7}, \alpha_{7}$, and $\sigma$ be as in Examples 4.3 and 6.1. The quadratic construction on $\left(f_{7}, f_{7}^{\sigma}\right)$ yields a three-parameter family

$$
\left(\mathcal{X}: y_{1}^{2}=f_{7}\left(x_{1}\right)^{2}+s_{1} f_{7}\left(x_{1}\right)+s_{2}, \mathcal{Y}: y_{2}^{2}=f_{7}^{\sigma}\left(x_{2}\right)^{2}+s_{1} f_{7}^{\sigma}\left(x_{2}\right)+s_{2}\right)
$$

of pairs of hyperelliptic curves of genus 6 defined over $\mathbb{Q}\left(\alpha_{7}\right)$. Specializing $\mathcal{X}$ at $\left(s_{1}, s_{2}, t\right)=(1,0,1)$ and reducing modulo a prime of $\mathbb{Q}\left(\alpha_{7}\right)$ over 13 , we obtain a 
curve $\bar{X}$ over $\mathbb{F}_{13^{2}}$. The Weil polynomial of $J_{\bar{X}}$ is irreducible, and corresponds to the Weil coefficients

$$
w_{1}=-16, w_{2}=-46, w_{3}=3496, w_{4}=-36993, w_{5}=-464728, w_{6}=13747140 .
$$

Applying Lemma 3.1, we see that $J_{\bar{X}}$ is absolutely simple. Hence, the generic fibre of $\mathcal{J}_{\mathcal{X}}$ is absolutely simple.

The correspondence $V\left(y_{1}-y_{2}, A_{7}\left(x_{1}, x_{2}\right)\right)$ on $\mathcal{X} \times_{T} \mathcal{Y}$ induces a homomorphism $\phi: \mathcal{J}_{\mathcal{X}} \rightarrow \mathcal{J}_{\mathcal{Y}}$. We find that

$$
D_{X, Y}(\phi)=\left(\begin{array}{rrrrrr}
\alpha_{7} & 0 & 0 & 0 & 0 & 0 \\
0 & \alpha_{7} & 0 & 0 & 0 & 0 \\
-\left(2 \alpha_{7}+1\right) t & 0 & \alpha_{7}^{\sigma} & 0 & 0 & 0 \\
-\left(\alpha_{7}+4\right) t & -2\left(\alpha_{7}+4\right) t & 0 & \alpha_{7} & 0 & 0 \\
7\left(\alpha_{7}+2\right) t^{2} & -2\left(2 \alpha_{7}+1\right) t & 3\left(\alpha_{7}^{\sigma}+4\right) t & 0 & \alpha_{7}^{\sigma} & 0 \\
7\left(\alpha_{7}^{\sigma}+4\right) t^{2} & -7\left(2 \alpha_{7}-3\right) t^{2} & 3\left(\alpha_{7}^{\sigma}+4\right) t & 4\left(2 \alpha_{7}^{\sigma}+1\right) t & 0 & \alpha_{7}^{\sigma}
\end{array}\right) .
$$

Since $D_{Y, X}\left(\phi^{\dagger}\right)=D_{X, Y}(\phi)^{\sigma}$, we have

$$
D_{X, X}\left(\phi^{\dagger} \phi\right)=D_{X, Y}(\phi) D_{Y, X}\left(\phi^{\dagger}\right)=2 I_{6},
$$

so $\phi^{\dagger} \circ \phi=[2]_{\mathcal{J}_{\mathcal{X}}}$; hence $\operatorname{ker} \phi \cong(\mathbb{Z} / 2 \mathbb{Z})^{6}$ by Lemma 3.2. The image of $\mathcal{J}_{\mathcal{X}}$ in $\mathcal{A}_{6}$ is three-dimensional by Lemma 4.2 and Torelli's theorem. We conclude that $\phi$ is a three-dimensional family of $(\mathbb{Z} / 2 \mathbb{Z})^{6}$-isogenies of (generically) absolutely simple Jacobians, thus proving Theorem 1.1 for the third row of the table.

Example 6.3. Let $f_{11}, A_{11}, \alpha_{11}$, and $\sigma$ be as in Example 4.4. The linear construction on $\left(f_{11}, f_{11}^{\sigma}\right)$, yields a one-parameter family

$$
\left(\mathcal{X}: y_{1}^{2}=f_{11}\left(x_{1}\right)+s, \mathcal{Y}: y_{2}^{2}=f_{11}^{\sigma}\left(x_{2}\right)+s\right)
$$

of pairs of hyperelliptic curves of genus 5 over $\mathbb{Q}\left(\alpha_{11}\right)$. Specializing $\mathcal{X}$ at $s=0$ and reducing modulo a prime of $\mathbb{Q}\left(\alpha_{11}\right)$ over 7 , we obtain a curve $\bar{X}$ over $\mathbb{F}_{49}$. The Weil polynomial of $J_{\bar{X}}$ is irreducible, and corresponds to the Weil coefficients

$$
w_{1}=12, w_{2}=28, w_{3}=-152, w_{4}=3652, w_{5}=53722 .
$$

Applying Lemma 3.1, we see that $J_{\bar{X}}$ is absolutely simple. Hence, the generic fibre of $\mathcal{J}_{\mathcal{X}}$ is absolutely simple.

The correspondence $V\left(A_{11}\left(x_{1}, x_{2}\right), y_{1}-y_{2}\right)$ on $\mathcal{X} \times_{T} \mathcal{Y}$ induces a homomorphism $\phi: \mathcal{J}_{\mathcal{X}} \rightarrow \mathcal{J}_{\mathcal{Y}}$. We find that

$$
D_{X, Y}(\phi)=\left(\begin{array}{rrrrr}
\alpha_{11} & 0 & 0 & 0 & 0 \\
0 & \alpha_{11}^{\sigma} & 0 & 0 & 0 \\
\alpha_{11}+6 & 0 & \alpha_{11} & 0 & 0 \\
0 & 0 & 0 & \alpha_{11} & 0 \\
-3\left(5 \alpha_{11}-3\right) & 4\left(2 \alpha_{11}+1\right) & 3\left(\alpha_{11}+6\right) & 0 & \alpha_{11}
\end{array}\right) .
$$

Since $D_{Y, X}\left(\phi^{\dagger}\right)=D_{X, Y}(\phi)^{\sigma}$, we have $D_{X}\left(\phi^{\dagger} \phi\right)=3 I_{5}$, so $\phi^{\dagger} \circ \phi=[3]_{\mathcal{J}_{\mathcal{X}}}$; hence $\operatorname{ker} \phi \cong(\mathbb{Z} / 3 \mathbb{Z})^{5}$ by Lemma 3.2. The image of $\mathcal{J}_{\mathcal{X}}$ in $\mathcal{A}_{5}$ is one-dimensional by Lemma 4.2 and Torelli's theorem. We conclude that $\phi$ is a one-dimensional family of $(\mathbb{Z} / 3 \mathbb{Z})^{5}$-isogenies of (generically) absolutely simple Jacobians, thus proving Theorem 1.1 for the second row of the table.

Example 6.4. Let $f_{11}, A_{11}, \alpha_{11}$, and $\sigma$ be as in Examples 4.4 and 6.3. The quadratic construction on $\left(f_{11}, f_{11}^{\sigma}\right)$ yields a two-parameter family

$$
\left(\mathcal{X}: y_{1}^{2}=f_{11}\left(x_{1}\right)^{2}+s_{1} f_{11}\left(x_{1}\right)+s_{2}, \mathcal{Y}: y_{2}^{2}=f_{11}^{\sigma}\left(x_{2}\right)^{2}+s_{1} f_{11}(x)+s_{2}\right)
$$

of pairs of hyperelliptic curves of genus 10 defined over $\mathbb{Q}\left(\alpha_{11}\right)$. Specializing $\mathcal{X}$ at $\left(s_{1}, s_{2}\right)=(1,0)$ and reducing modulo a prime of $\mathbb{Q}\left(\alpha_{11}\right)$ over 7 , we obtain a curve $\bar{X}$ over $\mathbb{F}_{49}$. The Weil polynomial of $J_{\bar{X}}$ is irreducible, and corresponds to the Weil 
TABLE 6. Weil polynomial coefficients for Example 6.4

\begin{tabular}{|r|l|l|l|l|l|r|l|r|l|}
\hline$i$ & $w_{i}$ & $i$ & $w_{i}$ & $i$ & $w_{i}$ & $i$ & $w_{i}$ & $i$ & $w_{i}$ \\
\hline \hline 1 & 0 & 2 & -16 & 3 & 196 & 4 & 2024 & 5 & 2484 \\
6 & 35208 & 7 & 127220 & 8 & 10074824 & 9 & 24089728 & 10 & -169499466 \\
\hline
\end{tabular}

coefficients listed in Table 6. Applying Lemma 3.1, we see that $J_{\bar{X}}$ is absolutely simple. Hence, the generic fibre of $\mathcal{J}_{\mathcal{X}}$ is absolutely simple.

The correspondence $V\left(A_{11}\left(x_{1}, x_{2}\right), y_{1}-y_{2}\right)$ on $\mathcal{X} \times_{T} \mathcal{Y}$ induces a homomorphism $\phi: \mathcal{J}_{\mathcal{X}} \rightarrow \mathcal{J}_{\mathcal{Y}}$. The $10 \times 10$ matrix $D_{X, Y}(\phi)$ is lower-triangular, with diagonal entries

$$
\alpha_{11}, \alpha_{11}^{\sigma}, \alpha_{11}, \alpha_{11}, \alpha_{11}, \alpha_{11}^{\sigma}, \alpha_{11}^{\sigma}, \alpha_{11}^{\sigma}, \alpha_{11}, \alpha_{11}^{\sigma},
$$

each of which is an element of norm 3 (we omit the other entries for lack of space). We therefore have

$$
D_{X, Y}(\phi) D_{Y, X}\left(\phi^{\dagger}\right)=D_{X, Y}(\phi) D_{X, Y}(\phi)^{\sigma}=3 I_{10},
$$

so $\phi^{\dagger} \phi=[3]_{\mathcal{X}}$; hence $\operatorname{ker} \phi \cong(\mathbb{Z} / 3 \mathbb{Z})^{10}$ by Lemma 3.2 . The image of $\mathcal{J}_{\mathcal{X}}$ in $\mathcal{A}_{10}$ is two-dimensional by Lemma 4.2 and Torelli's theorem. We conclude that $\phi$ is a two-dimensional family of $(\mathbb{Z} / 3 \mathbb{Z})^{10}$-isogenies of (generically) absolutely simple Jacobians, thus proving Theorem 1.1 for the sixth row of the table.

Example 6.5. Let $f_{13}, A_{13}, \alpha_{13}, \beta_{13}$ and $\sigma$ be as in Example 4.5. The linear construction on $\left(f_{13}, f_{13}^{\sigma}\right)$ yields a two-parameter family

$$
\left(\mathcal{X}: y_{1}^{2}=f_{13}\left(x_{1}\right)+s, \mathcal{Y}: y_{2}^{2}=f_{13}^{\sigma}\left(x_{2}\right)+s\right)
$$

of pairs of hyperelliptic curves of genus 6 . Specializing $\mathcal{X}$ at $(s, t)=(1,0)$, we obtain the curve $X$ of Example 5.1 with $n=13$, which has an absolutely simple Jacobian; hence the generic fibre of $\mathcal{J}_{\mathcal{X}}$ is absolutely simple.

The correspondence $V\left(A_{13}\left(x_{1}, x_{2}\right), y_{1}-y_{2}\right)$ on $\mathcal{X} \times_{T} \mathcal{Y}$ induces a homomorphism $\phi: \mathcal{J}_{\mathcal{X}} \rightarrow \mathcal{J}_{\mathcal{Y}}$. The $6 \times 6$ matrix $D_{X, Y}(\phi)$ is lower-triangular; if we set $e_{1}:=$ $\left(\beta_{13}-4\right) \alpha_{13}+2$ and $e_{2}:=\alpha+1$, then the diagonal entries of $D_{X, Y}(\phi)$ are

$$
e_{1}, e_{2}, e_{1}, e_{1}^{\sigma}, e_{2}, e_{2},
$$

each of which is an element of norm 3 in $\mathbb{Q}\left(\beta_{13}\right)$. (we omit the other entries for lack of space). We therefore have

$$
D_{X, Y}(\phi) D_{Y, X}\left(\phi^{\dagger}\right)=D_{X, Y}(\phi) D_{X, Y}(\phi)=3 I_{6},
$$

so $\phi^{\dagger} \circ \phi=[3]_{\mathcal{J}_{\mathcal{X}}}$; hence $\operatorname{ker} \phi \cong(\mathbb{Z} / 3 \mathbb{Z})^{6}$ by Lemma 3.2 . The image of $\mathcal{J}_{\mathcal{X}}$ in $\mathcal{A}_{6}$ is one-dimensional by Lemma 4.2 and Torelli's theorem. We conclude that $\phi$ is a one-dimensional family of $(\mathbb{Z} / 3 \mathbb{Z})^{6}$-isogenies of (generically) absolutely simple Jacobians, thus proving Theorem 1.1 for the fourth row of the table.

Example 6.6. Let $f_{13}, A_{13}, \alpha_{13}, \beta_{13}$ and $\sigma$ be as in Examples 4.5 and 6.5. The quadratic construction on $\left(f_{13}, f_{13}^{\sigma}\right)$ yields a three-parameter family

$$
\left(\mathcal{X}: y_{1}^{2}=f_{13}\left(x_{1}\right)^{2}+s_{1} f_{13}\left(x_{1}\right)+s_{2}, \mathcal{Y}: y_{2}^{2}=f_{13}^{\sigma}\left(x_{2}\right)^{2}+s_{1} f_{13}^{\sigma}\left(x_{2}\right)+s_{2}\right)
$$

of pairs of hyperelliptic curves of genus 12 defined over $\mathbb{Q}\left(\alpha_{13}\right)$. Specializing $\mathcal{X}$ at $\left(s_{1}, s_{2}, t\right)=(1,1,1)$ and reducing modulo a prime of $\mathbb{Q}\left(\alpha_{13}\right)$ over 5 , we obtain a curve $\bar{X}$ over $\mathbb{F}_{5^{4}}$. The Weil polynomial of $J_{\bar{X}}$ is irreducible, and corresponds to the Weil coefficients listed in Table 7. Applying Lemma 3.1, we see that $J_{\bar{X}}$ is absolutely simple. Hence, the generic fibre of $\mathcal{J}_{\mathcal{X}}$ is absolutely simple.

The correspondence $\left.V\left(A_{13}\left(x_{1}, x_{2}\right), y_{1}-y_{2}\right)\right)$ on $\mathcal{X} \times_{T} \mathcal{Y}$ induces a homomorphism $\phi: \mathcal{J}_{\mathcal{X}} \rightarrow \mathcal{J}_{\mathcal{Y}}$. The $12 \times 12$ matrix $D_{X, Y}(\phi)$ is lower-triangular, with diagonal entries

$$
e_{1}, \quad e_{2}, \quad e_{1}, \quad e_{1}^{\sigma}, \quad e_{2}, \quad e_{2}, \quad e_{2}^{\sigma}, \quad e_{2}^{\sigma}, \quad e_{1}, \quad e_{1}^{\sigma}, \quad e_{2}^{\sigma}, \quad e_{1}^{\sigma}
$$


TABLE 7. Weil polynomial coefficients for Example 6.6

\begin{tabular}{|r|l|r|l|r|l|r|l|}
\hline$i$ & $w_{i}$ & $i$ & $w_{i}$ & $i$ & $w_{i}$ & $i$ & $w_{i}$ \\
\hline \hline 1 & 20 & 4 & 351295 & 7 & 67298212 & 10 & -49877419547660 \\
2 & -230 & 5 & 1293764 & 8 & 137879604915 & 11 & 1975333453052116 \\
3 & -9232 & 9 & -1707055263168 & 6 & -204257742 & 12 & 119629530410659866 \\
\hline
\end{tabular}

(with $e_{1}$ and $e_{2}$ defined as in Example 6.5), each of which is an element of norm 3 in $\mathbb{Q}\left(\beta_{13}\right)$. We therefore have

$$
D_{X, Y}(\phi) D_{Y, X}\left(\phi^{\dagger}\right)=D_{X, Y}(\phi) D_{X, Y}(\phi)^{\sigma}=3 I_{12},
$$

so $\phi^{\dagger} \phi=[3]_{\mathcal{X}}$; hence $\operatorname{ker} \phi \cong(\mathbb{Z} / 3 \mathbb{Z})^{12}$ by Lemma 3.2 . The image of $\mathcal{J}_{\mathcal{X}}$ in $\mathcal{A}_{12}$ is three-dimensional by Lemma 4.2 and Torelli's theorem. We conclude that $\phi$ is a three-dimensional family of $(\mathbb{Z} / 3 \mathbb{Z})^{12}$-isogenies of (generically) absolutely simple Jacobians, thus proving Theorem 1.1 for the eighth row of the table.

Example 6.7. Let $f_{15}, A_{15}, \alpha_{15}$, and $\sigma$ be as in Example 4.6. The linear construction on $\left(f_{15},-f_{15}^{\sigma}\right)$ yields a two-parameter family

$$
\left(\mathcal{X}: y_{1}^{2}=f_{15}\left(x_{2}\right)+s, \mathcal{Y}: y_{2}^{2}=-f_{15}^{\sigma}\left(x_{2}\right)+s\right)
$$

of pairs of hyperelliptic curves of genus 7 defined over $\mathbb{Q}\left(\alpha_{15}\right)$. Specializing $\mathcal{X}$ at $(s, t)=(0,1)$ and reducing modulo a prime of $\mathbb{Q}\left(\alpha_{15}\right)$ over 17 , we obtain a curve $\bar{X}$ over $\mathbb{F}_{17}$. The Weil polynomial $\chi$ of $J_{\bar{X}}$ is irreducible, and corresponds to the Weil coefficients

$w_{1}=0, w_{2}=-4, w_{3}=-30, w_{4}=158, w_{5}=972, w_{6}=-2264, w_{7}=-18434$.

Applying Lemma 3.1, we see that $J_{\bar{X}}$ is absolutely simple. Hence, the generic fibre of $\mathcal{J}_{\mathcal{X}}$ is absolutely simple.

The correspondence $V\left(A_{15}\left(x_{1}, x_{2}\right), y_{1}-y_{2}\right)$ on $\mathcal{X} \times_{T} \mathcal{Y}$ induces a homomorphism $\phi: \mathcal{J}_{\mathcal{X}} \rightarrow \mathcal{J}_{\mathcal{Y}}$. The $7 \times 7$ matrix $D_{X, Y}(\phi)$ is lower-triangular with diagonal entries

$$
\alpha_{15}^{\sigma}, \alpha_{15}^{\sigma},-2, \alpha_{15}^{\sigma}, 2,2,-\alpha_{15},
$$

each of which is an element of norm 4 (we omit the other entries for lack of space). We therefore find

$$
D_{X, Y}(\phi) D_{Y, X}\left(\phi^{\dagger}\right)=D_{X, Y}(\phi) D_{X, Y}(\phi)^{\sigma}=4 I_{7},
$$

so $\phi^{\dagger} \circ \phi=[4]_{\mathcal{J}_{\mathcal{X}}}$. Specializing at $(s, t)=(1,0)$ and reducing modulo a prime over 31 , we obtain curves $\bar{X}: \bar{y}_{1}^{2}=\bar{x}_{1}^{15}+1$ and $\bar{Y}: \bar{y}_{2}^{2}=-\bar{x}_{2}^{15}+1$, together with an isogeny $\bar{\phi}: J_{\bar{X}} \rightarrow J_{\bar{Y}}$ induced by $V\left(\bar{A}\left(\bar{x}_{1}, \bar{x}_{2}\right), \bar{y}_{1}-\bar{y}_{2}\right) \subset \bar{X} \times \bar{Y}$, where

$$
\bar{A}=\bar{x}_{1}^{7}+14 \bar{x}_{1}^{6} \bar{x}_{2}-2 \bar{x}_{1}^{5} \bar{x}_{2}^{2}+19 \bar{x}_{1}^{4} \bar{x}_{2}^{3}+15 \bar{x}_{1}^{3} \bar{x}_{2}^{4}-2 \bar{x}_{1}^{2} \bar{x}_{2}^{5}+18 \bar{x}_{1} \bar{x}_{2}^{6}+\bar{x}_{2}^{7} .
$$

The polynomials $x_{1}^{15}+1$ and $-x_{2}^{15}+1$ both split completely over $\mathbb{F}_{31}$. Applying Lemmas 3.4 and 3.3 , we see that $\operatorname{ker} \bar{\phi} \cong(\mathbb{Z} / 4 \mathbb{Z})^{4} \times(\mathbb{Z} / 2 \mathbb{Z})^{6}$. The image of $\mathcal{J}_{\mathcal{X}}$ in $\mathcal{A}_{7}$ is two-dimensional by Lemma 4.2 and Torelli's theorem. We conclude that $\phi$ is a two-dimensional family of $(\mathbb{Z} / 4 \mathbb{Z})^{4} \times(\mathbb{Z} / 2 \mathbb{Z})^{6}$-isogenies of (generically) absolutely simple Jacobians, thus proving Theorem 1.1 for the fifth row of the table.

Example 6.8. Let $f_{15}, A_{15}, \alpha_{15}$, and $\sigma$ be as in Examples 4.6 and 6.7. The quadratic construction on $\left(f_{15},-f_{15}^{\sigma}\right)$ yields a three-parameter family

$$
\left(\mathcal{X}: y_{1}^{2}=f_{15}\left(x_{2}\right)^{2}+s_{1} f_{15}\left(x_{2}\right)+s_{2}, \mathcal{Y}: y_{2}^{2}=f_{15}^{\sigma}\left(x_{2}\right)^{2}-s_{1} f_{15}^{\sigma}\left(x_{2}\right)+s_{2}\right)
$$

of pairs of hyperelliptic curves of genus 14 defined over $\mathbb{Q}\left(\alpha_{15}\right)$. Specializing at $\left(s_{1}, s_{2}, t\right)=(1,1,1)$ and reducing modulo a prime of $\mathbb{Q}\left(\alpha_{15}\right.$ over 17 , we obtain a curve $\bar{X}$ over $\mathbb{F}_{17}$. The Weil polynomial $\chi$ of $J_{\bar{X}}$ is irreducible, and corresponds 
TABLE 8. Weil polynomial coefficients for Example 6.8

\begin{tabular}{|r|l|r|l|r|l|r|l|r|l|}
\hline$i$ & $w_{i}$ & $i$ & $w_{i}$ & $i$ & $w_{i}$ & $i$ & $w_{i}$ & $i$ & $w_{i}$ \\
\hline \hline 1 & -4 & 4 & -73 & 7 & 5874 & 10 & 1252762 & 13 & 80232390 \\
2 & 15 & 5 & 1000 & 8 & 29004 & 11 & -1381092 & 14 & -230738522 \\
3 & -6 & 6 & -1182 & 9 & 22810 & 12 & 8168424 & & \\
\hline
\end{tabular}

TABLE 9. Weil polynomial coefficients for Example 6.9

\begin{tabular}{|r||l|l|l|l|l|l|l|l|l|l|}
\hline$i$ & 1 & 2 & 3 & 4 & 5 & 6 & 7 & 8 & 9 & 10 \\
\hline$w_{i}$ & 4 & 36 & 272 & 1268 & 6492 & 28540 & 142200 & 453284 & 1065612 & 17399206 \\
\hline
\end{tabular}

to the Weil coefficients listed in Table 8. Applying Lemma 3.1, we see that $J_{\bar{X}}$ is absolutely simple. Hence, the generic fibre of $\mathcal{J}_{\mathcal{X}}$ is absolutely simple.

The correspondence $V\left(A_{31}\left(x_{1}, x_{2}\right), y_{1}-y_{2}\right)$ on $\mathcal{X} \times_{T} \mathcal{Y}$ induces a homomorphism $\phi: \mathcal{J}_{\mathcal{X}} \rightarrow \mathcal{J}_{\mathcal{Y}}$. The $14 \times 14$ matrix $D_{X, Y}(\phi)$ is lower-triangular with diagonal entries

$$
\alpha_{15}^{\sigma}, \alpha_{15}^{\sigma},-2, \alpha_{15}^{\sigma}, 2,2,-\alpha_{15}, \alpha_{15}^{\sigma},-2,-2,-\alpha_{15}, 2,-\alpha_{15}, \alpha_{15},
$$

each of which is an element of norm 4 (we omit the other entries for lack of space.) We therefore find

$$
D_{X, Y}(\phi) D_{Y, X}\left(\phi^{\dagger}\right)=D_{X, Y}(\phi) D_{X, Y}(\phi)^{\sigma}=4 I_{14},
$$

so $\phi^{\dagger} \circ \phi=[4]_{\mathcal{J}_{X}}$. Specializing at $\left(s_{1}, s_{2}, t\right)=(0,-1,0)$ and reducing modulo a prime over 31 , we obtain curves $\bar{X}: \bar{y}_{1}^{2}=\bar{x}_{1}^{30}-1$ and $\bar{Y}: \bar{y}_{2}^{2}=\bar{x}_{2}^{30}-1$, together with an isogeny $\bar{\phi}: J_{\bar{X}} \rightarrow J_{\bar{Y}}$ induced by $V\left(\bar{A}\left(\bar{x}_{1}, \bar{x}_{2}\right), \bar{y}_{1}-\bar{y}_{2}\right) \subset \bar{X} \times \bar{Y}$, where

$$
\bar{A}=\bar{x}_{1}^{7}+14 \bar{x}_{1}^{6} \bar{x}_{2}-2 \bar{x}_{1}^{5} \bar{x}_{2}^{2}+19 \bar{x}_{1}^{4} \bar{x}_{2}^{3}+15 \bar{x}_{1}^{3} \bar{x}_{2}^{4}-2 \bar{x}_{1}^{2} \bar{x}_{2}^{5}+18 \bar{x}_{1} \bar{x}_{2}^{6}+\bar{x}_{2}^{7} .
$$

The polynomial $x_{i}^{30}-1$ splits completely over $\mathbb{F}_{31}$. Applying Lemmas 3.4 and 3.3, we see that $\operatorname{ker} \bar{\phi} \cong(\mathbb{Z} / 4 \mathbb{Z})^{9} \times(\mathbb{Z} / 2 \mathbb{Z})^{10}$. The image of $\mathcal{J}_{\mathcal{X}}$ in $\mathcal{A}_{14}$ is threedimensional by Lemma 4.2 and Torelli's theorem. We conclude that $\phi$ is a family of $(\mathbb{Z} / 4 \mathbb{Z})^{9} \times(\mathbb{Z} / 2 \mathbb{Z})^{10}$-isogenies of (generically) absolutely simple Jacobians, thus proving Theorem 1.1 for the ninth row of the table.

Example 6.9. Let $f_{21}, A_{21}, \alpha_{21}$, and $\sigma$ be as in Example 4.7. The linear construction on $\left(f_{21}, f_{21}^{\sigma}\right)$ yields a one-parameter family

$$
\left(\mathcal{X}: y_{1}^{2}=f_{21}\left(x_{1}\right)+s, \mathcal{Y}: y_{2}^{2}=f_{21}^{\sigma}\left(x_{2}\right)+s\right)
$$

of pairs of hyperelliptic curves of genus 10 over $\mathbb{Q}\left(\alpha_{21}\right)$. Specializing $\mathcal{X}$ at $s=0$ and reducing modulo a prime of $\mathbb{Q}\left(\alpha_{21}\right)$ over 5 , we obtain a curve $\bar{X}$ over $\mathbb{F}_{25}$. The Weil polynomial $\chi$ of $J_{\bar{X}}$ is irreducible, and corresponds to the Weil coefficients listed in Table 9. Applying Lemma 3.1, we see that $J_{\bar{X}}$ is absolutely simple. Hence, the generic fibre of $\mathcal{J}_{\mathcal{X}}$ is absolutely simple.

The correspondence $V\left(A_{21}\left(x_{1}, x_{2}\right), y_{1}-y_{2}\right)$ on $\mathcal{X} \times_{T} \mathcal{Y}$ induces a homomorphism $\phi: \mathcal{J}_{\mathcal{X}} \rightarrow \mathcal{J}_{\mathcal{Y}}$. The $10 \times 10$ matrix $D_{X, Y}(\phi)$ is lower-triangular; if we set $e=\left(\alpha_{21}^{\sigma}\right)^{2}$, then the diagonal entries of $D_{X, Y}(\phi)$ are

$$
\left(\alpha_{21}^{\sigma}\right)^{2},\left(\alpha_{21}^{\sigma}\right)^{2},-\left(\alpha_{21}^{\sigma}\right)^{2},\left(\alpha_{21}^{\sigma}\right)^{2}, \alpha_{21}^{2},-\left(\alpha_{21}^{\sigma}\right)^{2}, \alpha_{21} \alpha_{21}^{\sigma},\left(\alpha_{21}^{\sigma}\right)^{2},-\alpha_{21}^{2}, \alpha_{21}^{2},
$$

each of which is an element of norm 4 (we omit the other entries for lack of space). We therefore have

$$
D_{X, Y}(\phi) D_{Y, X}\left(\phi^{\dagger}\right)=D_{X, Y}(\phi) D_{X, Y}(\phi)^{\sigma}=4 I_{10},
$$

so $\phi^{\dagger} \circ \phi=[4]_{\mathcal{J}_{\mathcal{X}}}$. Specializing at $s=425$ and reducing modulo a prime over 599 , we obtain curves $\bar{X}$ and $\bar{Y}$ and an isogeny $\bar{\phi}: J_{\bar{X}} \rightarrow J_{\bar{Y}}$ over $\mathbb{F}_{599}$. Applying 
TABLE 10. Weil polynomial coefficients for Example 6.10

\begin{tabular}{|r|l|r|l|r|l|r|l|r|l|}
\hline$i$ & $w_{i}$ & $i$ & $w_{i}$ & $i$ & $w_{i}$ & $i$ & $w_{i}$ & $i$ & $w_{i}$ \\
\hline \hline 1 & -4 & 5 & -1616 & 9 & -431556 & 13 & -83783104 & 17 & -12690445996 \\
2 & 13 & 6 & 5919 & 10 & 1564993 & 14 & 294134355 & 18 & 43906230241 \\
3 & -74 & 7 & -24382 & 11 & -5699656 & 15 & -1000833886 & 19 & -144999550062 \\
4 & 403 & 8 & 105299 & 12 & 22091457 & 16 & 3592033583 & 20 & 476625334323 \\
\hline
\end{tabular}

Lemmas 3.4 and 3.3 , we find $\left.\operatorname{ker} \bar{\phi}\right|_{J_{\bar{X}}[2]} \cong(\mathbb{Z} / 2 \mathbb{Z})^{11}$, so $\operatorname{ker} \phi \cong(\mathbb{Z} / 4 \mathbb{Z})^{9} \times(\mathbb{Z} / 2 \mathbb{Z})^{2}$. The image of $\mathcal{J}_{\mathcal{X}}$ in $\mathcal{A}_{10}$ is one-dimensional by Lemma 4.2 and Torelli's theorem. We conclude that $\phi$ is a one-dimensional family of $(\mathbb{Z} / 4 \mathbb{Z})^{9} \times(\mathbb{Z} / 2 \mathbb{Z})^{2}$-isogenies of (generically) absolutely simple Jacobians, thus proving Theorem 1.1 for the seventh row of the table.

Example 6.10. Let $f_{21}, A_{21}, \alpha_{21}$, and $\sigma$ be as in Examples 4.7 and 6.9. The quadratic construction on $\left(f_{21}, f_{21}^{\sigma}\right)$ yields a two-parameter family

$$
\left(\mathcal{X}: y_{1}^{2}=f_{21}\left(x_{1}\right)^{2}+s_{1} f_{21}\left(x_{1}\right)+s_{2}, \mathcal{Y}: y_{2}^{2}=f_{21}^{\sigma}\left(x_{2}\right)^{2}+s_{1} f_{21}^{\sigma}\left(x_{2}\right)+s_{2}\right)
$$

of pairs of hyperelliptic curves of genus 10 defined over $\mathbb{Q}\left(\alpha_{21}\right)$. Specializing $\mathcal{X}$ at $\left(s_{1}, s_{2}\right)=(1,1)$ and reducing modulo a prime of $\mathbb{Q}\left(\alpha_{21}\right)$ over 11 , we obtain a curve $\bar{X}$ over $\mathbb{F}_{11}$. The Weil polynomial $\chi$ of $J_{\bar{X}}$ is irreducible, and corresponds to the Weil coefficients listed in Table 10. Applying Lemma 3.1, we see that $J_{\bar{X}}$ is absolutely simple. Hence, the generic fibre of $\mathcal{J}_{\mathcal{X}}$ is absolutely simple.

The correspondence $C=V\left(A_{21}\left(x_{1}, x_{2}\right), y_{1}-y_{2}\right)$ on $\mathcal{X}_{20} \times_{T} \mathcal{Y}_{20}$ induces a homomorphism $\phi: \mathcal{J}_{\mathcal{X}} \rightarrow \mathcal{J}_{\mathcal{Y}}$. The $20 \times 20$ matrix $D_{X, Y}(\phi)$ is a lower-triangular; if we set $e:=-\left(\alpha_{21}+1\right)$, then the diagonal entries of $D_{X, Y}(\phi)$ are

$\left(\alpha_{21}^{\sigma}\right)^{2},\left(\alpha_{21}^{\sigma}\right)^{2},-\left(\alpha_{21}^{\sigma}\right)^{2},\left(\alpha_{21}^{\sigma}\right)^{2}, \alpha_{21}^{2},-\left(\alpha_{21}^{\sigma}\right)^{2}, \alpha_{21} \alpha_{21}^{\sigma},\left(\alpha_{21}^{\sigma}\right)^{2},-\alpha_{21}^{2}, \alpha_{21}^{2}$,

$\left(\alpha_{21}^{\sigma}\right)^{2},-\left(\alpha_{21}^{\sigma}\right)^{2}, \alpha_{21}^{2}, \alpha_{21} \alpha_{21}^{\sigma},-\alpha_{21}^{2},\left(\alpha_{21}^{\sigma}\right)^{2}, \alpha_{21}^{2},-\alpha_{21}^{2}, \alpha_{21}^{2}, \alpha_{21}^{2}$,

each of which is an element of norm 4 (we omit the other entries for lack of space). We therefore find

$$
D_{X, Y}(\phi) D_{Y, X}\left(\phi^{\dagger}\right)=D_{X, Y}(\phi) D_{X, Y}(\phi)^{\sigma}=4 I_{20},
$$

so $\phi^{\dagger} \circ \phi=[4]_{\mathcal{J}_{\mathcal{X}}}$. Specializing at $\left(s_{1}, s_{2}\right)=(1,6)$ and reducing at a prime over 29 , we obtain curves $\bar{X}$ and $\bar{Y}$ and an isogeny $\bar{\phi}: J_{\bar{X}} \rightarrow J_{\bar{Y}}$ over $\mathbb{F}_{29}$. Applying Lemmas 3.4 and 3.3 , we see that $\operatorname{ker} \bar{\phi} \cong(\mathbb{Z} / 4 \mathbb{Z})^{19} \times(\mathbb{Z} / 2 \mathbb{Z})^{2}$. The image of $\mathcal{J}_{\mathcal{X}}$ in $\mathcal{A}_{20}$ is two-dimensional by Lemma 4.2 and Torelli's theorem. We conclude that $\phi$ is a two-dimensional family of $(\mathbb{Z} / 4 \mathbb{Z})^{19} \times(\mathbb{Z} / 2 \mathbb{Z})^{2}$-isogenies of (generically) absolutely simple Jacobians, thus proving Theorem 1.1 for the eleventh row of the table.

Example 6.11. Let $f_{31}, A_{31}, \alpha_{31}, \beta_{31}$, and $\sigma$ be as in Example 4.8. The linear construction on $\left(f_{31}, f_{31}^{\sigma}\right)$ yields a one-parameter family

$$
\left(\mathcal{X}: y_{1}^{2}=f_{31}\left(x_{1}\right)+s, \mathcal{Y}: y_{2}^{2}=f_{31}^{\sigma}\left(x_{2}\right)+s\right)
$$

of pairs of hyperelliptic curves of genus 15 over $\mathbb{Q}\left(\alpha_{31}\right)$. Specializing $\mathcal{X}$ at $s=0$ and reducing modulo a prime of $\mathbb{Q}\left(\alpha_{31}\right)$ over 5 , we obtain a curve $\bar{X}$ over $\mathbb{F}_{5^{3}}$. The Weil polynomial of $J_{\bar{X}}$ is irreducible, and corresponds to the Weil coefficients listed in Table 11. The Jacobian $J_{\bar{X}}$ is absolutely simple by Lemma 3.1; hence the generic fibre of $\mathcal{J}_{\mathcal{X}}$ is absolutely simple.

The correspondence $C=V\left(A\left(x_{1}, x_{2}\right), y_{1}-y_{2}\right)$ on $\mathcal{X} \times_{T} \mathcal{Y}$ induces a homomorphism $\phi: \mathcal{J}_{\mathcal{X}} \rightarrow \mathcal{J}_{\mathcal{Y}}$. The $15 \times 15$ matrix $D_{X, Y}(\phi)$ is lower-triangular; if we set $e_{1}:=-\left(\left(\beta_{31}^{2}-9 \beta_{31}+14\right) \alpha_{31}+4 \beta_{31}-16\right) / 4, e_{2}:=-\left(\left(\beta_{31}-6\right) \alpha_{31}+\beta_{31}^{2}-8 \beta_{31}+8\right) / 2$, and $e_{3}:=\alpha_{31}-\beta_{31}+4$, then the diagonal entries of $D_{X, Y}(\phi)$ are

$$
e_{1}, e_{1}, e_{2}, e_{1}, e_{3}, e_{2}, e_{2}^{\sigma}, e_{1}, e_{3}, e_{3}, e_{3}^{\sigma}, e_{2}, e_{3}^{\sigma}, e_{2}^{\sigma}, e_{1}^{\sigma},
$$


TABLE 11. Weil polynomial coefficients for Example 6.11

\begin{tabular}{|r|l|r|l|r|l|r|l|}
\hline$i$ & $w_{i}$ & $i$ & $w_{i}$ & $i$ & $w_{i}$ & $i$ & $w_{i}$ \\
\hline \hline 1 & 25 & 5 & 146470 & 9 & -5019303477 & 13 & 17625044970092 \\
2 & 447 & 6 & -1950824 & 10 & 9095279162 & 14 & -265293278436450 \\
3 & 5046 & 7 & -61460901 & 11 & 544453054742 & 15 & -4448335615035972 \\
4 & 42930 & 8 & -750851497 & 12 & 5818130546490 & & \\
\hline
\end{tabular}

TABLE 12. Weil polynomial coefficients for Example 6.12

\begin{tabular}{|r|l|r|l|}
\hline$i$ & $w_{i}$ & $i$ & $w_{i}$ \\
\hline \hline 1 & 86 & 14 & 2538874803438283085247 \\
2 & 3451 & 15 & 75551657032201511555544 \\
3 & 87828 & 16 & 2132291122470015060842077 \\
4 & 1643613 & 17 & 58726738607409603792625818 \\
5 & 43045482 & 18 & 1634122583940469502202897151 \\
6 & 1781887735 & 19 & 48450321094461320825161410124 \\
7 & 76936315232 & 20 & 1504867060985705824450391696293 \\
8 & 3105710470069 & 21 & 45345655631250765718117003095430 \\
9 & 102095895729754 & 22 & 1270533776275133738442812562176203 \\
10 & 2779643454835731 & 23 & 34526697723237826449755783511899672 \\
11 & 71233879362094240 & 24 & 956449237011673888073922827627521777 \\
12 & 2193677250388156081 & 25 & 26767220948731629452685495358053131182 \\
13 & 77619720346267760370 & 26 & 757441695740127512275452904130818491239 \\
\hline 27 & 21123226183916202851140834209673472022292 \\
28 & 575803060349811307421020344590821665754597 \\
29 & 15365239367923178818677513358710798508553810 \\
30 & 408015365744689122150660893862413952306834751 \\
\hline
\end{tabular}

each of which is an element of norm 8 in $\mathbb{Q}\left(\beta_{31}\right)$ (we omit the other entries for lack of space). We therefore find

$$
D_{X, Y}(\phi) D_{Y, X}\left(\phi^{\dagger}\right)=D_{X, Y}(\phi) D_{X, Y}(\phi)^{\sigma}=8 I_{15},
$$

so $\phi^{\dagger} \circ \phi=[8]_{\mathcal{J}_{X}}$. Specializing at $s=0$ and reducing modulo a prime over 47 , we obtain curves $\bar{X}$ and $\bar{Y}$ and an isogeny $\bar{\phi}: J_{\bar{X}} \rightarrow J_{\bar{Y}}$ over $\mathbb{F}_{47}$. Applying Lemmas 3.4 and 3.3, we find $\operatorname{ker} \bar{\phi} \cong(\mathbb{Z} / 8 \mathbb{Z})^{5} \times(\mathbb{Z} / 4 \mathbb{Z})^{10} \times(\mathbb{Z} / 4 \mathbb{Z})^{10}$. The image of $\mathcal{J}_{\mathcal{X}}$ in $\mathcal{A}_{15}$ is one-dimensional by Lemma 4.2 and Torelli's theorem. We conclude that $\phi$ is a one-dimensional family of $(\mathbb{Z} / 8 \mathbb{Z})^{5} \times(\mathbb{Z} / 4 \mathbb{Z})^{10} \times(\mathbb{Z} / 4 \mathbb{Z})^{10}$-isogenies of (generically) absolutely simple Jacobians, thus proving Theorem 1.1 for the tenth row of the table.

Example 6.12. Let $f_{31}, A_{31}, \alpha_{31}, \beta_{31}$, and $\sigma$ be as in Examples 4.8 and 6.11. The quadratic construction on $\left(f_{31}, f_{31}^{\sigma}\right)$ yields a two-parameter family

$$
\left(\mathcal{X}: y_{1}^{2}=f_{31}\left(x_{1}\right)^{2}+s_{1} f_{31}\left(x_{1}\right)+s_{2}, \mathcal{Y}: y_{2}^{2}=f_{31}^{\sigma}\left(x_{1}\right)^{2}+s_{1} f_{31}^{\sigma}\left(x_{2}\right)+s_{2}\right)
$$

of pairs of hyperelliptic curves of genus 30 defined over $\mathbb{Q}\left(\alpha_{31}\right)$. Specializing $\mathcal{X}$ at $\left(s_{1}, s_{2}\right)=(1,2)$ and reducing modulo a prime of $\mathbb{Q}\left(\alpha_{31}\right)$ over 3 , we obtain a curve $\bar{X}$ over $\mathbb{F}_{3^{6}}$. The Weil polynomial of $J_{\bar{X}}$ is irreducible, and corresponds to the Weil coefficients listed in Table 12. The Jacobian $J_{\bar{X}}$ is absolutely simple by Lemma 3.1; hence the generic fibre of $\mathcal{J}_{\mathcal{X}}$ is absolutely simple.

The correspondence $C=V\left(A_{31}\left(x_{1}, x_{2}\right), y_{1}-y_{2}\right)$ on $\mathcal{X} \times_{T} \mathcal{Y}$ induces a homomorphism $\phi: \mathcal{J}_{\mathcal{X}} \rightarrow \mathcal{J}_{\mathcal{Y}}$. The $30 \times 30$ matrix $D_{X, Y}(\phi)$; is lower-triangular with diagonal 
entries

$$
\begin{aligned}
& e_{1}, e_{1}, e_{2}, e_{1}, e_{3}, e_{2}, e_{2}^{\sigma}, e_{1}, e_{3}, e_{3}, e_{3}^{\sigma}, e_{2}, e_{3}^{\sigma}, e_{2}^{\sigma}, e_{1}^{\sigma}, \\
& e_{1}, e_{2}, e_{3}, e_{2}^{\sigma}, e_{3}, e_{3}^{\sigma}, e_{3}^{\sigma}, e_{1}^{\sigma}, e_{2}, e_{2}^{\sigma}, e_{3}^{\sigma}, e_{1}^{\sigma}, e_{2}^{\sigma}, e_{1}^{\sigma}, e_{1}^{\sigma}
\end{aligned}
$$

(where $e_{1}, e_{2}$, and $e_{3}$ are defined as in Example 6.11), each of which is an element of norm 8 in $\mathbb{Q}\left(\beta_{31}\right)$ (we omit the other entries for lack of space). We therefore have

$$
D_{X, Y}(\phi) D_{Y, X}\left(\phi^{\dagger}\right)=D_{X, Y}(\phi) D_{X, Y}(\phi)^{\sigma}=8 I_{30},
$$

so $\phi^{\dagger} \circ \phi=[8]_{\mathcal{J}_{\mathcal{X}}}$. Specializing at $\left(s_{1}, s_{2}\right)=(4,9)$ and reducing modulo a prime over 47 , we obtain curves $\bar{X}$ and $\bar{Y}$ and an isogeny $\bar{\phi}: J_{\bar{X}} \rightarrow J_{\bar{Y}}$ over $\mathbb{F}_{47}$. Applying Lemmas 3.4 and 3.3, we find $\operatorname{ker} \bar{\phi} \cong(\mathbb{Z} / 8 \mathbb{Z})^{11} \times(\mathbb{Z} / 4 \mathbb{Z})^{19} \times(\mathbb{Z} / 4 \mathbb{Z})^{19}$. The image of $\mathcal{J}_{\mathcal{X}}$ in $\mathcal{A}_{30}$ is two-dimensional by Lemma 4.2 and Torelli's theorem. We conclude that $\phi$ is a two-dimensional family of $(\mathbb{Z} / 8 \mathbb{Z})^{11} \times(\mathbb{Z} / 4 \mathbb{Z})^{19} \times(\mathbb{Z} / 4 \mathbb{Z})^{19}$-isogenies of (generically) absolutely simple Jacobians, thus proving Theorem 1.1 for the twelfth row of the table.

\section{REFERENCES}

[1] Ch. Birkenhake and H. Lange, Complex abelian varieties (second edition), Grundlehren der mathematischen Wissenschaften 302, Springer-Verlag Berlin, 2004.

[2] W. Bosma, J. J. Cannon, and C. Playoust, The Magma algebra system. I. The user language, J. Symbolic Comput. 24(3-4) (1997), 235-265

[3] W. Bosma, J. J. Cannon, et. al., Handbook of Magma Functions, School of Mathematics and Statistics, University of Sydney (1995)

[4] J. W. S. Cassels, Factorization of polynomials in several variables, Proceedings of the 15th Scandinavian Congress, Oslo 1968, Springer Lecture Notes in Mathematics 118 (1970), 1-17

[5] P. Cassou-Nogues and J.-M. Couveignes, Factorisations explicities de $g(y)-h(z)$, Acta Arithmetica 87 (1999), no. 4, 291-317

[6] C.-L. Chai and F. Oort, A note on the existence of absolutely simple Jacobians, Journal of Pure and Applied Algebra 155 (2001), 115-120

[7] W. Feit, Automorphisms of symmetric balanced incomplete block designs, Math. Z. 118 (1970), 40-49

[8] W. Feit, On symmetric balanced incomplete block designs with doubly transitive automorphism groups, Journal of Combinatorial Theory (A) 14 (1973), 221-247

[9] W. Feit, Some consequences of the classification of finite simple groups, Proceedings of Symposia in Pure Math. 37 (1980), 175-181

[10] M. Fried, On a conjecture of Schur, Michigan Math. J. 17 (1970), 41-55

[11] M. Fried, The field of definition of function fields and a problem in the reducibility of polynomials in two variables, Illinois J. Math. 17 (1973), 128-146

[12] M. Fried, Exposition on an arithmetic-group theoretic connection via Riemann's existence theorem, Proceedings of Symposia in Pure Math. 37 (1980), 571-602

[13] M. C. Harrison, Implementation of Kedlaya's algorithm, in [2, 3]

[14] M. Hindry and J. Silverman, Diophantine geometry: an introduction, GTM 201, Springer (2000).

[15] E. W. Howe and H. J. Zhu, On the existence of absolutely simple abelian varieties of a given dimension over an arbitrary field, J. Number Theory 92 (2002), 139-163

[16] K. S. Kedlaya, Counting points on hyperelliptic curves using Monsky-Washnitzer cohomology, J. Ramanujan Math. Soc. 16 (2001), no. 4, 323-338

[17] D. R. Kohel and B. A. Smith, Efficiently computable endomorphisms for hyperelliptic curves, in Algorithmic number theory: proceedings of ANTS-VII, LNCS 4076 (2006) 495-509

[18] G. Kux, Construction of algebraic correspondences between hyperelliptic function fields using Deuring's theory, Ph.D. thesis, Universität Kaiserslautern (2004)

[19] D. Lehavi and C. Ritzenthaler: An explicit formula for the arithmetic geometric mean in genus 3, Experimental Math. 16 (2007) 421-440

[20] R. Lidl, G. L. Mullen and G. Turnwald, Dickson polynomials, Pitman monographs and surveys in pure and applied mathematics 65, Longman Scientific and Technical (1993)

[21] J.-F. Mestre, Couples de jacobiennes isogénes de courbes hyperelliptiques de genre arbitraire. Preprint arXiv:0902.3470v1 [math.AG]

[22] J. S. Milne, Abelian Varieties, In G. Cornell J. H. Silverman (ed.), Arithmetic Geometry, Springer (1986) 
[23] G. Shimura, Abelian varieties with complex multiplication and modular functions, Princeton mathematical series 46, Princeton University Press (1998)

[24] B. Smith, Explicit endomorphisms and correspondences, Ph.D. thesis, University of Sydney (2006)

[25] B. Smith, Isogenies and the discrete logarithm problem in Jacobians of genus 3 hyperelliptic curves. In N. Smart (ed.), EUROCRYPT 2008, LNCS 4965 (2008) 163-180

[26] W. Tautz, J. Top, and A. Verberkmoes, Explicit hyperelliptic curves with real multiplication and permutation polynomials, Canad. J. Math. 43 (1991), no. 5, 1055-1064

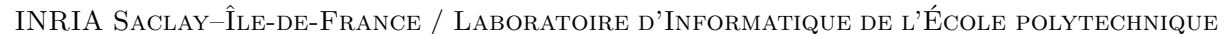
(LIX), 91128 Palaiseau Cedex, France

E-mail address: smith@lix.polytechnique.fr 